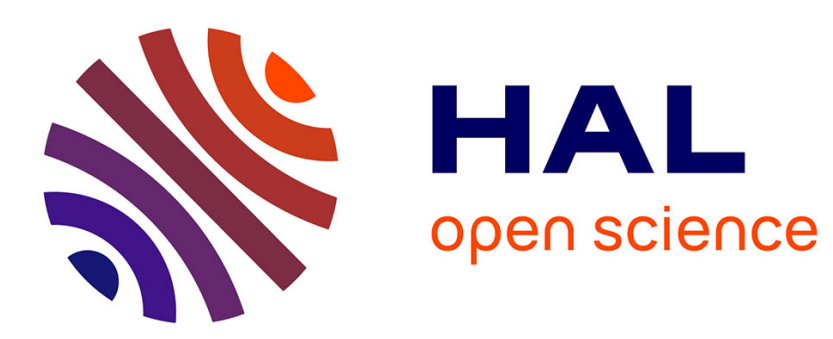

\title{
Passive separation control using a self-adaptive hairy coating
}

\author{
Julien Favier, Antoine Dauptain, Davide Basso, Alessandro Bottaro
}

\section{To cite this version:}

Julien Favier, Antoine Dauptain, Davide Basso, Alessandro Bottaro. Passive separation control using a self-adaptive hairy coating. Journal of Fluid Mechanics, 2009, 627, pp.451 - 483. hal-01073967

\section{HAL Id: hal-01073967 https://hal.science/hal-01073967}

Submitted on 11 Oct 2014

HAL is a multi-disciplinary open access archive for the deposit and dissemination of scientific research documents, whether they are published or not. The documents may come from teaching and research institutions in France or abroad, or from public or private research centers.
L'archive ouverte pluridisciplinaire HAL, est destinée au dépôt et à la diffusion de documents scientifiques de niveau recherche, publiés ou non, émanant des établissements d'enseignement et de recherche français ou étrangers, des laboratoires publics ou privés. 


\title{
Passive separation control using a self-adaptive hairy coating
}

\author{
JULIEN FAVIER, ANTOINE DAUPTAIN $\dagger$, \\ DAVIDE BASSO AND ALESSANDRO BOTTARO
}

DICAT, Universita di Genova, Via Montallegro 1, 16145, Genova, Italy

A model of hairy medium is developed using a homogenized approach, and the fluid flow around a circular cylinder partially coated with hair is analyzed by means of numerical simulations. The capability of this coating to adapt to the surrounding flow is investigated, and its benefits are discussed in the context of separation control. This fluid-structure interaction problem is solved with a partitioned approach, based on the direct resolution of the Navier-Stokes equations together with a non-linear set of equations describing the dynamics of the coating. A volume force, arising from the presence of a cluster of hair, provides the link between the fluid and the structure problems. For the structure part, a subset of reference elements approximates the whole layer. The dynamics of these elements is governed by a set of equations based on the inertia, elasticity, interaction and losses effects of articulated rods. The configuration chosen is that of the two-dimensional flow past a circular cylinder at $R e=200$, a simple and well documented test case. Aerodynamics performances quantified by the Strouhal number, the drag and the maximum lift in the laminar unsteady regime are modified by the presence of the coating. A set of parameters corresponding to a realistic coating (length of elements, porosity, rigidity) is found, yielding an average drag reduction of $15 \%$ and a decrease of lift fluctuations by about $40 \%$, associated to a stabilization of the wake.

\section{Introduction}

The manipulation of fluid flows to bring about performance enhancements on air/water vehicles is a topic of growing interest in the fluid mechanics community. Besides the highly stimulating and fundamental problems raised by the control of the non-linear Navier-Stokes equations, flow control has a tremendous economical and ecological impact on society (see Gad-el-Hak (2000) for a detailed survey). In this context, it is particularly worthwhile to analyze swimming and flying animals, in order to import novel ideas into technological applications. Not surprisingly many efficient locomotion techniques are found in Nature, as they have survived the tests of evolution over millions of years and reached a high level of adaptation. One interesting example is represented by the feathers over the wings of birds. Even though it is difficult to monitor their dynamics due to the animal's rapid motion, they are believed to play a crucial role in the aerodynamics of birds. As mentioned in the excellent review on biological surface by Bechert et al. (1997) and Meyer et al. (2007), the pop-up of feathers observed on snapshots and movies of landing birds is probably relevant for the control of flow separation.

Several drag-reducing biological surfaces inspired by aquatic animals have also shown their efficiency:

- riblets are inspired by the skin of sharks (Bechert \& Bartenwerfer 1989; Luchini et al. 1991) and allow to reduce the shear stress compared to a smooth surface; they have been

$\dagger$ Present address: CERFACS, 42 Avenue Gaspard Coriolis, 31057 Toulouse Cedex 01, France. 
successfully tested on large airplanes (Viswanath 2002), although in-service application appears to be prevented by the need to replace the riblet film every two or three years;

- the presence of bumps on whale flippers can delay stall and thus enhance hydrodynamics manoeuvrability performances (van Nierop et al. 2008);

- the release of trapped air bubbles from the skin of a penguin appears to have an effect on the reduction of skin friction (Xu et al. 2002).

By looking at this short list it may appear that straightforward mimicry of nature might lead to novel and efficient technological applications. The task is however not so straightforward. In-depth understanding of physical mechanisms is required to manufacture efficient actuators since a biological skin is meant to handle multiple functions: for example the presence of mucus on the skin of fish may protect it against parasites and infections, and has a drag reducing function as well. Thus, direct imitation of the skin of fish in the effort to minimize drag might yield a sub-optimal solution, since the skin performs many other functions.

The so-called Gray paradox of the compliant skins of dolphins is a striking example of the difficulty to mimick a biological surface. It was believed that the impressive swimming ability of dolphins was due to the compliance of their skin, able to delay transition to turbulence and/or maintain a laminar boundary layer on the surface of the dolphin's body. Many studies were inspired by the original observations of Gray \& Sand (1936), starting with the theories of Benjamin (1960) and Landahl (1962), and later with the analyses of disturbances developing in boundary layers over compliant plates (Carpenter \& Garrad 1985, 1986). It is now clear that Gray's premises were flawed, as mentioned by Fish \& Lauder (2006) and confirmed recently by Hœpffner et al. (2008); the latter authors have shown that compliance can yield very large transient disturbance amplifications compared to smooth surfaces, potentially dangerous for the onset of turbulence. Fish \& Lauder (2006) have demonstrated that the drag reduction observed on dolphins is linked mostly to behavioral functions of the animal, mainly related to its breathing habits.

Coming back to the presence and function of feathers on the wings of birds, we aim here at making progress in understanding the effect of the feathers (or similar protuberances) on the aerodynamic performances. All birds have six different types of feathers covering their body, performing different tasks during flight. They are adapted to flight conditions, and used for many purposes, including to shape the wings, insulate and protect the animal's skin. This type of system is then clearly very complex to model, but the property of interest here is the ability of the wings to adapt to the surrounding flow to influence the aerodynamics ( $c f$. figure 1 ). The assumption that the raising of feathers during birds' landing phases plays a role in the increase of the lift coefficient of the wing has to be demonstrated. It is probable that this pop-up is not coincidental, but is due to a self-adaptation of birds' wings to the separated flow during landing, in order to control it. Outstanding questions are then: do the feathers act like classical slats on commercial airplanes wings which locally increase the angle of attack? Do they behave like vortex generators stabilizing the recirculation zone by redistributing energy? Is it more of a slowing down effect due to the suddenly popped-up porous fence or another effect affecting the stability of the boundary layer towards separation?

The physical mechanism is not clearly identified so far: although the impact on the flow is as yet undefined, we believe, along with Bechert et al. (1997), that the phenomenon is worth studying since there are indications that "these small feathers are important for the flight control of birds at high lift conditions during landing." Indeed, the control of the recirculation zone would explain the amazing manoeuvrability aptitudes of birds, experiencing high angles of attack with a perfect wing stability. Incidentally it has already 

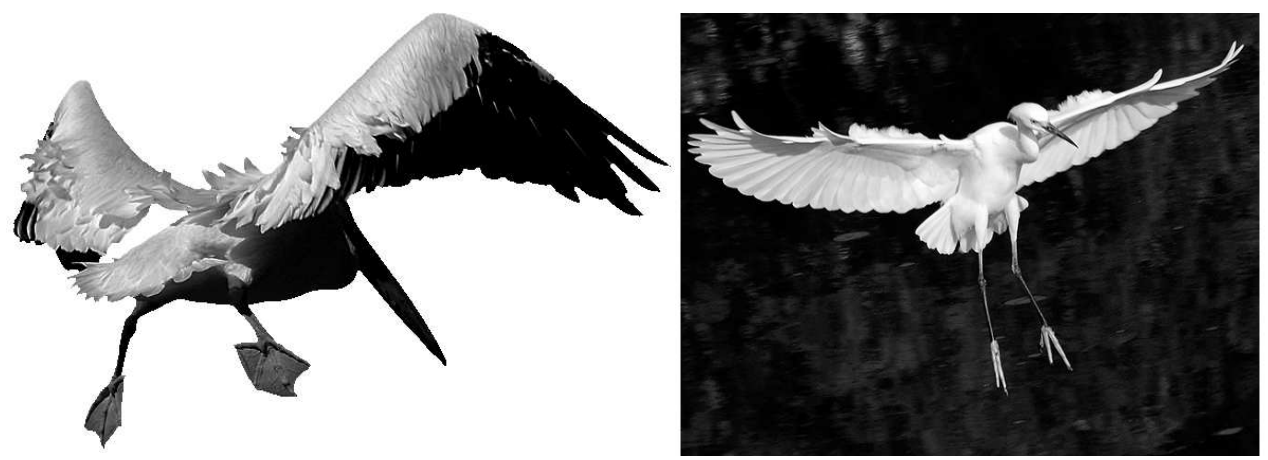

FiguRE 1. Raising of birds feathers, observed during landing phases. Left: snapshot of a pelican just before landing (thus gliding flight). Right: pop-up of feathers observed on the upper-side of the wings during the landing of an egret (courtesy of $\mathrm{J} \mathrm{R}$ Compton, http://www.jrcompton.com/birds/).

been found that a static porous layer can be used as a mean of boundary layer separation control (Bruneau \& Mortazavi 2008).

The study of the flow past hairy coatings finds many applications: for example in the study of thick bundles of immersed vegetation (Sukhodolova et al. 2004) and windexposed plants (De Langre 2008) in strong interaction with the surrounding fluid flows. Another possible application of porous fuzzy coatings is found in the realm of sports: for example the felt of a tennis ball plays an important role on the aerodynamics of the ball (Mehta \& Pallis 2001) and new techniques of digital imaging have recently been implemented by Steele et al. (2006) to properly assess the quality of the textile surface roughness, predict ball performances and develop acceptable wear limits. Finally, new concepts of sensors and actuators for flow control are based on tiny rod-like elements, whose deflection provides a measure of the wall shear stress (Brücker et al. 2005; Große \& Schröder 2008).

In this paper we build a simplified model of hairy coating, with the following "featherlike" characteristics:

- porous, since fluid can flow through the feathers; the non-homogenous character of the coating formed by the different types of feathers, more or less packed, is taken into account through a density parameter,

- non-isotropic, as fluid is oriented along a specific direction as it enters the layer, just like in realistic feathers,

- compliant, since the layer can bend and deform according to the surrounding flow. Such properties are those which appear to us as the most important in modeling birds' feathers. The possibility of shape adaptation of this wall coating is tested and analysed on a classical and academic configuration of separated flow: the motion around a twodimensional circular cylinder at Reynolds number $R e=200$. The numerical framework is presented to clearly illustrate the simulation procedures relative to fluid and structure parts, the assumptions on which the model is built, its perspectives of further applications and limitations.

As this domain of investigation is naturally related to the studies of flows through arrays of fibers, we will base our work on experimental, theoretical and numerical results on such configurations (Howells 1998; Koch \& Ladd 1997). Various models of different orders of approximation are built for the drag per unit length, as a function of the density of fibers, in Howells (1998). Different organizations of fibers (parallel, random) are assessed and estimates are made to take into account the effects of finite length, 


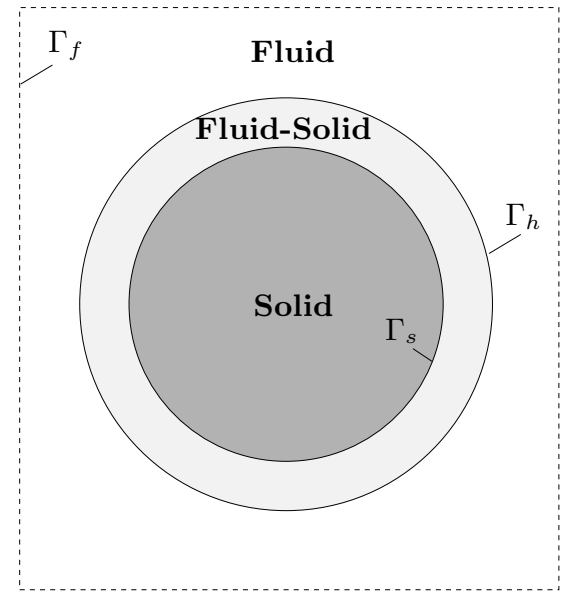

Figure 2. The three zones of the computational domain (not to scale).

curvature and neighbouring fibers interactions, leading to results in good agreement with experiments. These theoretical developements will be useful to derive an expression of the volume force used in this article.

Schematically, the domain of study is decomposed into three zones corresponding to a solid body, a surrounding fluid in motion and a mixed fluid-solid portion representing the hairy coating. In figure 2 the fluid area is included between the fixed boundary $\left(\Gamma_{f}\right)$ of the fluid domain, and the fixed boundary $\left(\Gamma_{s}\right)$ of the cylinder. The hairy layer between $\Gamma_{s}$ and the moving boundary $\Gamma_{h}$ is in interaction with the fluid. There is no mass exchange between fluid and solid domains and the temperature is assumed to be constant and uniform throughout.

The first sections of the article are dedicated to a description of the numerical treatment of the fluid and structure parts, and how the two-way coupling between the two is achieved. The application that follows refers to the control of the unsteady wake and it illustrates the potential of the approach. In the following the elements forming the coating will be referred to as pillars, hair, cilia, beams or fibers, always to mean the same thing.

\section{Fluid domain}

\subsection{Equations}

The simulation of the unsteady flow around a cylinder of diameter $D$ is performed by solving the discrete version of the incompressible Navier-Stokes equations in a twodimensional periodic domain. The equations are given below, with $\mathbf{U}$ Eulerian velocity, $p$ pressure, $\mu$ dynamic viscosity, $\rho$ density and $\mathbf{F}$ a volume force:

$$
\rho\left[\frac{\partial \mathbf{U}}{\partial t}+(\mathbf{U} \cdot \nabla) \mathbf{U}\right]=-\nabla p+\mu \nabla^{2} \mathbf{U}+\mathbf{F} ; \nabla \cdot \mathbf{U}=0 .
$$

A sketch of the domain over which equations (2.1) have been discretized is provided in figure 3. The Reynolds number is defined as $R e=\frac{\rho\left\|\mathbf{U}_{\infty}\right\| D}{\mu}$, with $\mathbf{U}_{\infty}$ the free stream velocity. The volume force $\mathbf{F}$ in (2.1) is decomposed into three contributions $\mathbf{F}=\mathbf{F}^{c}+\mathbf{F}^{b}+\mathbf{F}^{h}:$

(a) $\mathbf{F}^{c}$ is introduced to account for the presence of the solid cylinder, i.e. it renders 


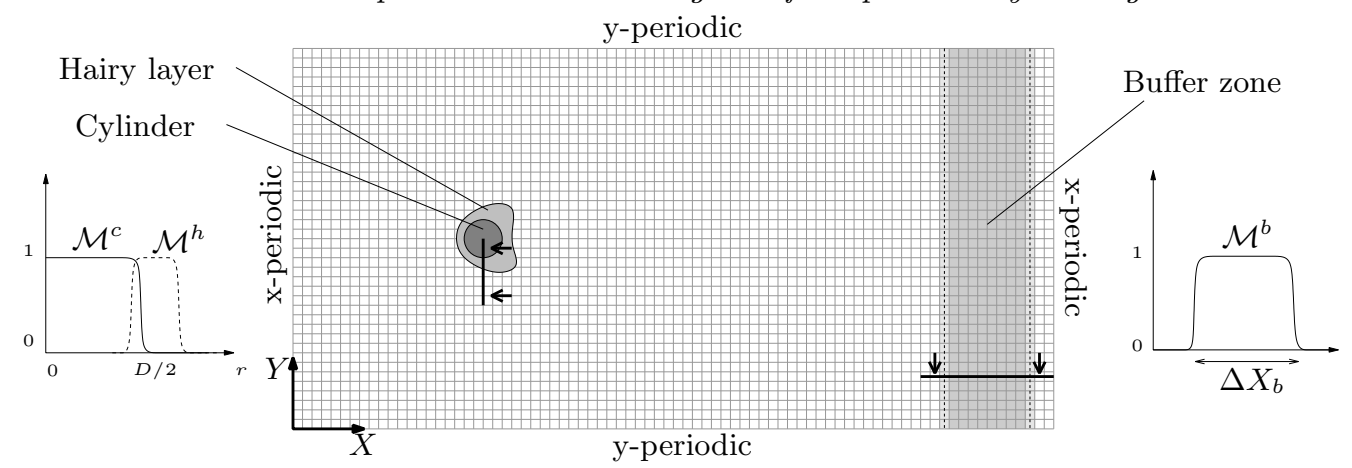

Figure 3. Computational domain of the fluid problem for the flow around a coated cylinder of diameter $D$. One cell over ten is represented on the mesh. The immersed boundary method is used for the solid cylinder through the function $\mathcal{M}^{c}$, the hairy layer through the function $\mathcal{M}^{h}$ and a buffer zone through the function $\mathcal{M}^{b}$.

equal to zero the fluid velocity inside the circular obstacle. This volume force is computed using the immersed boundary method described in Peskin (2002), i.e.

$$
\mathbf{F}^{c}=\mathcal{M}^{c}\left(\alpha^{c} \int_{t_{0}}^{t}(\mathbf{0}-\mathbf{U}) d t+\beta^{c}(\mathbf{0}-\mathbf{U})\right) .
$$

$\mathcal{M}^{c}$ is a non-dimensional scalar field equal to one inside the cylinder, zero outside, as shown in figure 3. Appropriate values of the positive constants $\alpha^{c}$ and $\beta^{c}$ are found to be respectively 1 and $6 / \Delta t$, with $\Delta t$ the time step of the computations. With this set of parameters, the velocity within the cylinder section is always such that $\int_{V_{1}}\|\mathbf{U}\| /\left\|\mathbf{U}_{\infty}\right\| \mathrm{d} x \mathrm{~d} y<10^{-5}$, where the volume of integration $V_{1}$ is the volume of the cylinder per unit depth.

(b) A buffer zone of thickness $\Delta X_{b}$ is imposed with a volume force $\mathbf{F}^{b}$ to damp the unsteady structures in the wake of the cylinder, before they reach the end of the domain. Since the domain is periodic, this buffer volume force is also used to ensure that the inflow speed is equal to $\mathbf{U}_{\infty}$ on the left-hand side of the domain (figure 3):

$$
\mathbf{F}^{b}=\mathcal{M}^{b}\left(\alpha^{b} \int_{t_{0}}^{t}\left(\mathbf{U}_{\infty}-\mathbf{U}\right) d t+\beta^{b}\left(\mathbf{U}_{\infty}-\mathbf{U}\right)\right) .
$$

$\mathcal{M}^{b}$ is equal to one inside the buffer layer, zero outside. Here $\alpha^{b}$ and $\beta^{b}$ are set to 0.8 and $15 / \Delta t$, such that the velocity at the exit of the buffer zone in a control volume $V_{2}$ of thickness $D$ is $\int_{V_{2}}\left(1-\|\mathbf{U}\| /\left\|\mathbf{U}_{\infty}\right\|\right) \mathrm{d} x \mathrm{~d} y<10^{-5}$.

(c) The hairy layer is imposed with a force $\mathbf{F}^{h}$, evaluated as the drag force past a cluster of tiny beams of various density and orientation $(c f . \S 3.1) . \mathbf{F}^{h}$ vanishes strictly outside the volume occupied by the coating.

For the three immersed boundary domains, it is necessary to smooth the edges of the filter functions by using a progressive interpolation. A hyperbolic tangent function is used on $\mathcal{M}^{b}$ for the buffer zone, and a distributed interpolation approach is employed for the cylinder and the hairy layer $\left(\mathcal{M}^{c}\right.$ and $\left.\mathcal{M}^{h}\right)$, following the methodology described in Dauptain et al. (2008).

\subsection{Resolution and convergence}

To solve (2.1), a finite difference formulation is used on a regular cartesian mesh made up by $800 \times 400$ cells in a $40 D \times 20 D$ domain; we have ensured that this resolution yields grid-converged results for the flow past a cylinder. Staggered flow variables are 

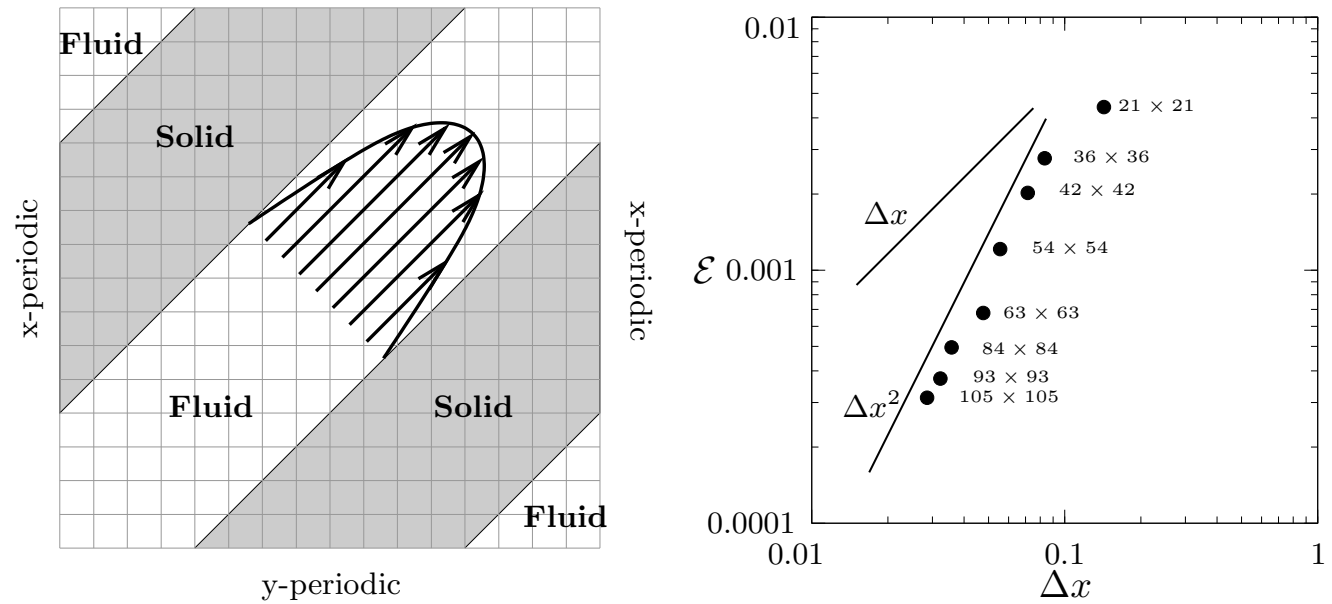

FiguRE 4. Spatial convergence of the fluid solver. Left: Domain geometry with fluid and solid parts non aligned with the mesh. Right: Norm of the error versus grid spacing.

used. The solver uses the explicit Adams-Bashforth scheme for the convective part, and the semi-implicit Crank-Nicolson method for the viscous part. The Poisson equation for the pressure and the implicit step are treated by the conjugate gradient method (due to the periodic boundary conditions the matrices involved are symmetric and positive definite). This method is second order in time and space. Moreover, the periodicity of the domain and the use of the immersed boundary method allow straightforward and accurate computations of the energy balance terms and of aerodynamic loads. The time scales of fluid and structures phenomena are comparable; a restrictive condition on the time step of the simulation is imposed to make sure that such phenomena are properly captured.

To validate the immersed boundary method, a convergence study is performed on a two dimensional Poiseuille flow (figure 4, left frame). The domain is a periodic square, and the walls are not aligned with the mesh, with an inclination of $45^{\circ}$. The error $\mathcal{E}$ plotted in figure 4 (right frame) is the norm of the difference between the theoretical profile $U^{\text {th }}$ and the velocity $U$ on a cross section of the duct at the computational nodes $i=1, \ldots, N$ :

$$
\mathcal{E}=\frac{1}{N U_{\max }} \sqrt{\sum_{i=1}^{N}\left(U_{i}^{t h}-U_{i}\right)^{2}} .
$$

Grids ranging from $21 \times 21$ to $105 \times 105$ are tested to check the global order of the solver. Figure 4 demonstrates second-order convergence of the spatial resolutions for grids finer than $36 \times 36$. As far as the laminar flow past a cylinder is concerned, the lift and drag coefficients found in the literature (He et al. 2000; Bergmann et al. 2005) are well reproduced (see §6.1) and this is sufficient evidence for the solver to be considered suitable for the present investigation.

\subsection{Communications with the structure part}

The link between the fluid and the structure problems is done via the volume force $\mathbf{F}^{h}$, either expressed in the fluid discretization space $\left(\mathbf{F}_{i j}^{h}\right)$ or in the structure discretization space $\left(\mathbf{F}_{k}^{h}\right)$. The state variables of the fluid equations ( $\mathbf{U}, p$ and $\left.\mathbf{F}\right)$ are discretized in the space of dimensions $N_{x} \times N_{y}\left(\mathbf{U}_{i j}, p_{i j}\right.$ and $\left.\mathbf{F}_{i j}\right)$. On the other hand, the dynamics of 
the hairy layer is described via the angular positions $\theta_{k}$ of each reference element, with $k=1, \ldots, N_{c}(c f .3 .2)$, corresponding to a discretization in a space of dimension $N_{c}$.

\section{Hairy domain}

The coating is a dense cluster of hair and is described with a homogenized approach, as a non-isotropic, compliant layer of variable porosity. The motion in time of the layer is modelled by a set of non-linear equations derived from the dynamical equilibrium of the system. The coupling with the fluid part is described hereafter.

\subsection{Homogenized drag model}

The interaction of the hairy medium with the flow is taken into account with an estimate of the drag force past the cluster of hair sketched in figure $5 \mathrm{a}$. This force per unit volume $\mathbf{F}^{h}$ is assumed to be decomposed into a normal and a tangential component:

- $\mathbf{F}_{t}^{h}$ estimated as the drag force past a cluster of very long thin cylinders aligned with the flow,

- $\mathbf{F}_{n}^{h}$ approximated by the drag force past a random cluster of cylinders orthogonal to the flow.

In order to evaluate these components, we introduce the packing density $\phi=\mathcal{V}_{\text {hair }} / \mathcal{V}_{\text {layer }}$, ratio of the volume occupied by the hair (solid) over the total sampling volume. This quantity varies continuously between 0 (no cilia) and 1 (solid) inside the layer. Another variable is defined inside the layer, the unit orientation vector $\mathbf{d}$, characterizing the direction of each element of the coating. Both of these variables are schematically shown in figure $5 \mathrm{~b}$.

In the fixed reference frame of the cylinder, we can define for any point $P$ belonging to a reference element, its associated velocity $\mathbf{V}^{h}$. The velocity in $P$ relative to a fiber-like element is thus $\mathbf{U}^{h}=\mathbf{U}-\mathbf{V}^{h}$. Its component tangent to the element is the projection $\mathbf{U}_{t}^{h}=\left(\mathbf{U}^{h} \cdot \mathbf{d}\right)$, while the normal component is $\mathbf{U}_{n}^{h}=\mathbf{U}^{h}-\mathbf{U}_{t}^{h}$. The drag force component tangential to each element of the coating is assumed to depend on $\phi$ and $\mathbf{U}_{t}^{h}$ :

$$
\left\|\mathbf{F}_{t}^{h}\right\|=f_{1}\left(\phi, R e_{t}^{h}\right) .
$$

A similar hypothesis is made for the normal component:

$$
\left\|\mathbf{F}_{n}^{h}\right\|=f_{2}\left(\phi, R e_{n}^{h}\right),
$$

with $R e_{t}^{h}$ and $R e_{n}^{h}$ the Reynolds numbers based on the element's diameter and the local fluid velocity: $R e_{t}^{h}=\frac{\left\|\mathbf{U}_{t}^{h}\right\| d_{h}}{\nu}$ and $R e_{n}^{h}=\frac{\left\|\mathbf{U}_{n}^{h}\right\| d_{h}}{\nu}$.

Each element of the coating is assumed to be a rigid fiber of circular cross-section, so that $\mathbf{F}_{n}^{h}$ corresponds to the force exerted by an array of random cylinders as sketched in figure 6 . It is quite complex to cope with the multiple interactions among the cylinder wakes when $R e$ becomes large, and the approximation of the normal contribution $\mathbf{F}_{n}^{h}$ is thus limited to moderate values of the Reynolds numbers $R e_{n}^{h}$, up to 180 . We use theoretical and empirical scaling models presented in Koch \& Ladd (1997) to estimate this contribution of the force, as a function of $R e_{n}^{h}$, and the packing density $\phi$. According to their results, it is acceptable to employ a linear function of $R e_{n}^{h}$ as:

$$
\frac{\mathcal{F}_{n}^{h}}{\mu\left\|\mathbf{U}_{n}^{h}\right\|}=c_{0}(\phi)+c_{1}(\phi) R e_{n}^{h},
$$


Fluid
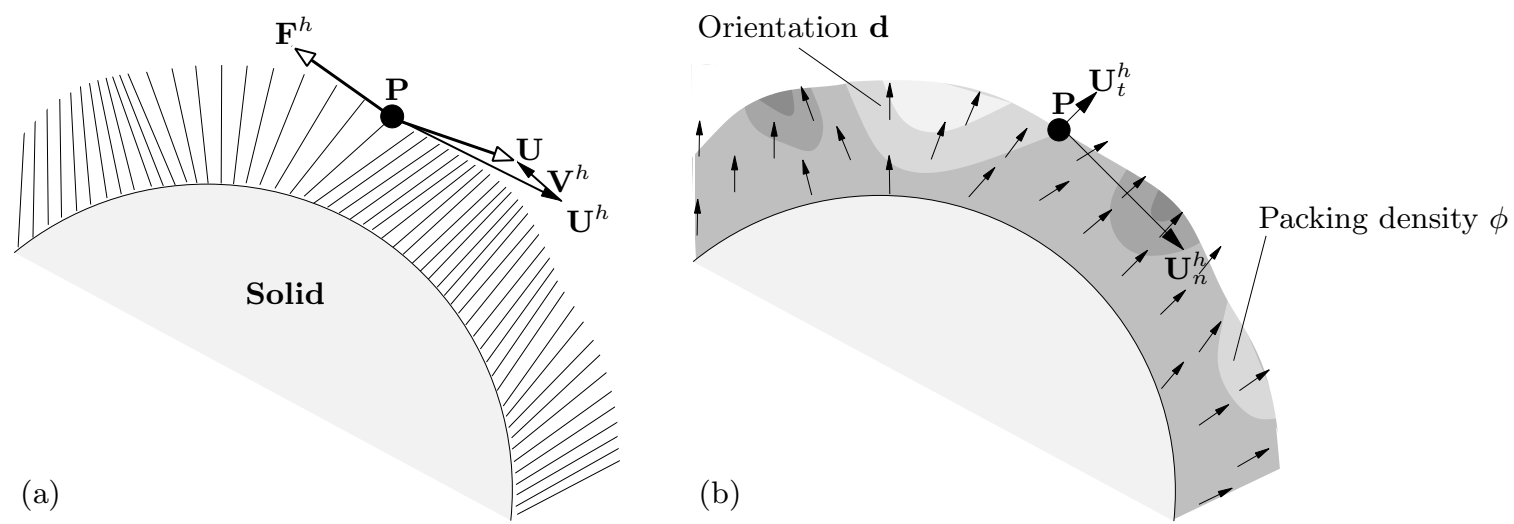

FiguRE 5. Homogenized model of the furry coating and sketch of the volume force $\mathbf{F}^{h}$ imposed on the fluid. (a) Hairy layer covering a cylinder and relative fluid velocity $\mathbf{U}^{h}$ at any point $\mathrm{P}$ of the layer, while the single element moves at velocity $\mathbf{V}^{h}$. (b) Gray scale of the packing density $\phi$ and vector $\mathbf{d}$ orientation of the elements within the hairy layer. At point $\mathrm{P}$, tangential and normal velocity components used to estimate the two components of $\mathbf{F}^{h}$ are displayed.

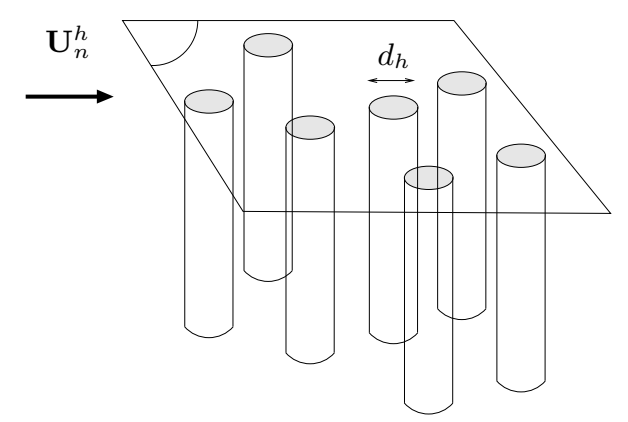

Figure 6. Configuration for the evaluation of the normal component of the drag force.

$\mathcal{F}_{n}^{h}$ being the normal force per cilium and unit length, such that $\left\|\mathbf{F}_{n}^{h}\right\|=\frac{4 \phi}{\pi d_{h}^{2}} \mathcal{F}_{n}^{h}$. The coefficient $c_{0}$ represents the Stokes' drag and $c_{1}$ is the inertial drag which governs the behavior in the larger-than-zero Re-regime. These two coefficients are function of the packing density $\phi$ of the porous medium corresponding to the configuration of figure 6 . The coefficient $c_{0}$ is estimated using Brinkman's law at $R e_{n}^{h}=0$ (Stokes' flow limit); $c_{1}$ is evaluated from the behaviour of the ratio $c_{1} / c_{0}$ over a wide range of $\phi$ given in Koch \& Ladd (1997). Their work also shows that this mixed theoretical/empirical approximation provides good agreement with the measurements.

For the non-dimensional tangential contribution $\frac{\mathcal{F}_{t}^{h}}{\mu\left\|\mathbf{U}_{t}^{h}\right\|}$, we derive an analytical expression, in the Stokes approximation, on the configuration of figure 7 corresponding to the axial flow between concentric cylinders. Using cylindrical coordinates and assuming a steady, fully developed axisymmetric flow $\left(\frac{\partial}{\partial z}=\frac{\partial}{\partial \theta}=\frac{\partial}{\partial t}=0\right)$ with $u_{r}=u_{\theta}=0$, we obtain an expression for the radial velocity around one cylinder between a fiber of radius 


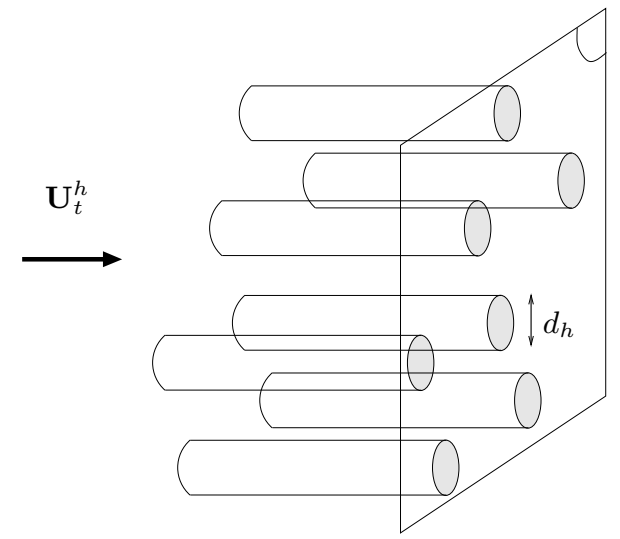

FiguRE 7. Configuration for the evaluation of the tangential component of the drag force.

$r_{1}=d_{h} / 2$ and the average pore of radius $r_{2}=r_{1} / \sqrt{\phi}$ :

$$
u(r)=\frac{F_{t}^{h}}{4 \mu}\left(2 r_{2}^{2} \ln \frac{r}{r_{1}}+r_{1}^{2}-r^{2}\right) .
$$

$F_{t}^{h}$ refers to the tangential component of the force, per unit volume. To introduce the incoming velocity $\left\|\mathbf{U}_{t}^{h}\right\|$ of the model, we then compute the mass flux $D$ of the flow passing through the cilia interspace $D=\rho \pi\left(r_{2}^{2}-r_{1}^{2}\right)\left\|\mathbf{U}_{t}^{h}\right\|(1-\phi)$, which is also equal to:

$$
D=\rho \int_{0}^{2 \pi} d \theta \int_{r_{1}}^{r_{2}} u(r) r d r
$$

After substituting $u(r)$ by its expression in (3.4). integrating and introducing the packing density $\phi=r_{1}^{2} / r_{2}^{2}$, the following expression is found:

$$
\frac{F_{t}^{h}}{\mu\left\|\mathbf{U}_{t}^{h}\right\|}=\frac{8 \phi(1-\phi)}{r_{1}^{2}\left(\phi-1+\frac{2}{\phi-1} \ln \phi-2\right)} .
$$

At this point, we recall that $F_{t}^{h}$ is a force per unit volume of fluid (which is equal to $\left.(1-\phi) \mathcal{V}_{\text {layer }}\right)$. The force per unit length of cilium is finally $\mathcal{F}_{t}^{h}=F_{t}^{h}(1-\phi) \pi r_{2}^{2}$, leading to

$$
\frac{\mathcal{F}_{t}^{h}}{\mu\left\|\mathbf{U}_{t}^{h}\right\|}=\frac{8 \pi(1-\phi)^{2}}{\phi-1+\frac{2}{\phi-1} \ln \phi-2} .
$$

Similarly to the normal contribution, $\mathcal{F}_{t}^{h}$ is the tangential force for one pillar and per unit length of the pillar, such that the volume force is $\left\|\mathbf{F}_{t}^{h}\right\|=\frac{4 \phi}{\pi d_{h}^{2}} \mathcal{F}_{t}^{h}$. We then assume for the tangential force the same scaling in Reynolds number as for the normal contribution, obtaining the behaviour displayed in figure 8 .

The angle that each element makes with the wall determines the relative magnitude of the tangential and normal velocity components. In the case of equivalent tangential and normal velocity magnitudes (i.e. when a fiber element is inclined at $45^{\circ}$ ), the tangential component of the force is typically much smaller that the normal one, as illustrated in figure 8. This difference in the two contributions tends to align the fibers with the flow. Once the elements are parallel to the flow, the normal component of the force becomes negligible with respect to the tangential one. These approximations of the forces are 


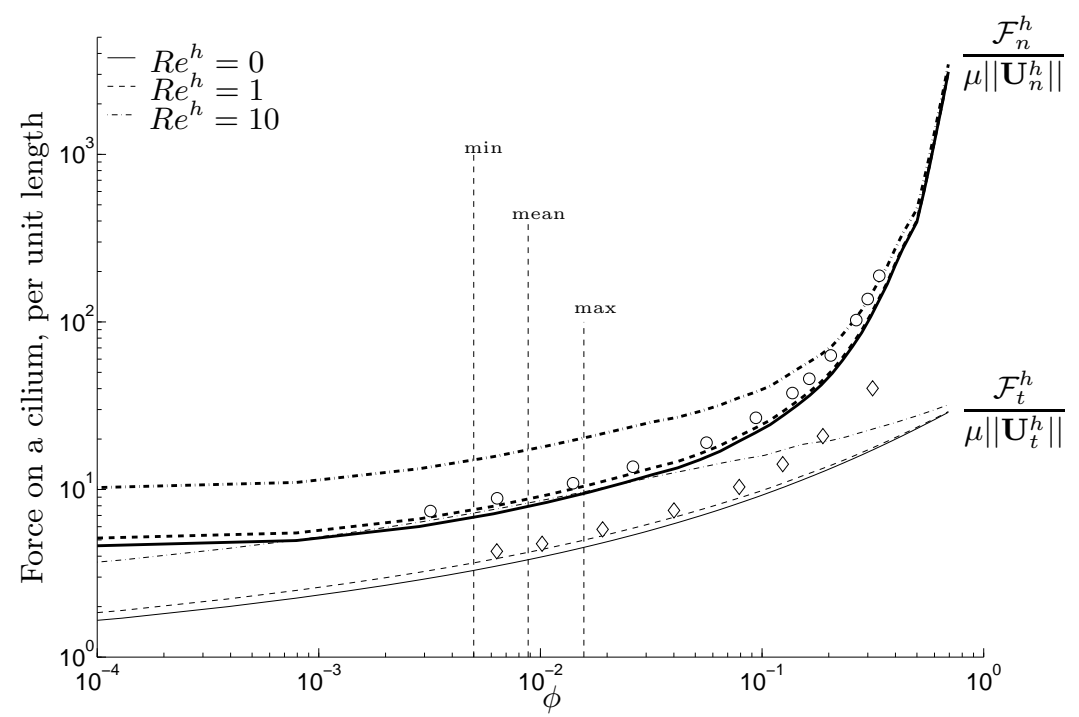

FIGURE 8. Dimensionless normal force $\frac{\mathcal{F}_{n}^{h}}{\mu\left\|\mathbf{U}_{n}^{h}\right\|}$ (bold lines) and tangential force $\frac{\mathcal{F}_{t}^{h}}{\mu\left\|\mathbf{U}_{t}^{h}\right\|}$ (thin lines) as functions of the packing density $\phi$. Three local Reynolds number $\left(R e_{t}^{h}\right.$ or $R e_{n}^{h}$ depending on the case) are considered (0,1 and 10), corresponding to the typical values encountered in the simulations. The theoretical values obtained by Howells (1998) for the longitudinal drag (diamond) and the normal drag (circles) in slow flow are shown. Vertical dashed lines indicate the minimum, maximum and mean values of $\phi$ used for the results of section $\S 6.3$.

in good agreement with the theoretical results by Howells (1998). Slight differences are observed on the tangential contribution, which we have computed analytically, mainly because the effect of the finite length of the cylinders is taken into account in Howells' model (cf. $\S 1)$.

Using this approach, the hairy layer is now described in terms of homogenized volume forces, allowing the communications with the fluid. The next paragraph deals with the dynamics of the coating, i.e. its evolution in time as a result of the forcing exerted by the fluid.

\subsection{Dynamical model for the hairy coating}

Modelling a realistic coating would normally require an extremely large number of fibers. The high number of degrees of freedom needed in this case is reduced using an homogenized approach: we thus assume that a few reference elements are sufficient to approximate the dynamics of the whole layer (figure 9a). The forces at play are supposed to be concentrated on reference cilia, which are equally spaced. A control volume surrounds each reference cilium. The model describing the dynamical evolution in time of the reference cilia is illustrated in figure 9b. Each element is a thin rigid pillar of length $l$ hinging on the wall, and its mass is placed in the middle of the rod. A pillar can oscillate in the plane around its equilibrium angle $\theta_{e q}$. To model the behaviour of the layer realistically, all cilia are linked to their immediate neighbours with a non-linear spring of stiffness $K_{s}$, which is active only when two elements become too close to one another (to counteract the compression effect). Another feature of the model, controlled by the parameter $K_{r}$, is used to mimick the tendency of each reference cilium to stay around $\theta_{e q}$. These mechanisms of interaction and rigidity are explained below; they are inspired by a model developed by Py et al. (2006) to study the effect of the wind over a flexible crop canopy. 

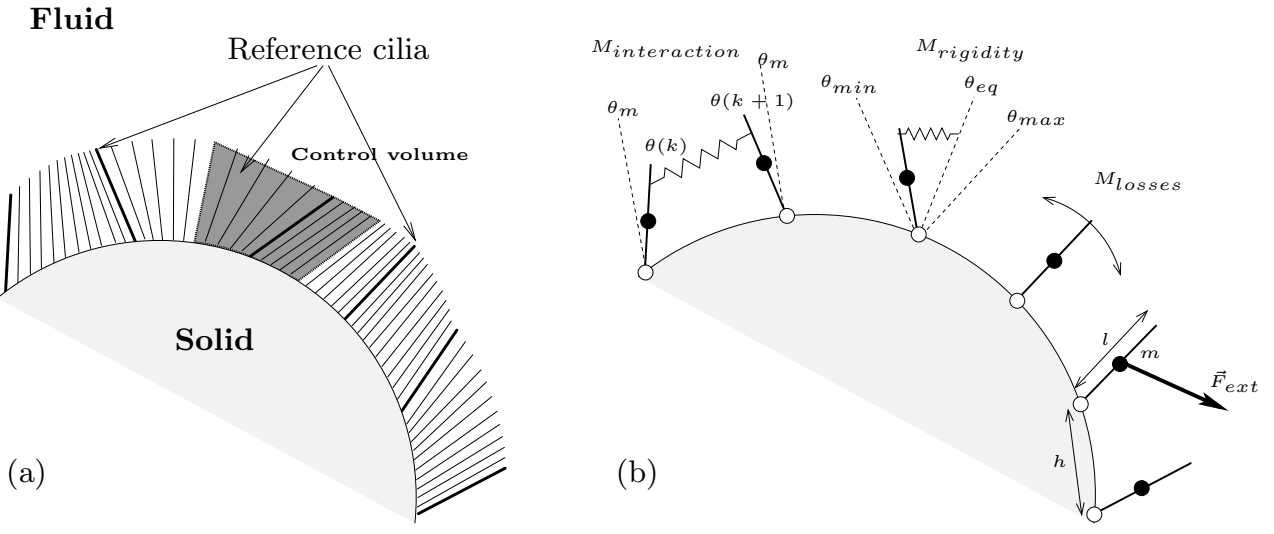

Figure 9. Model of the hairy coating. (a) Reference cilia (in bold) governing the dynamics of the layer. The control volume around one reference cilium is shown in gray. (b) Dynamical model of reference cilia based on mass, interaction, inertial, losses and rigidity effects.
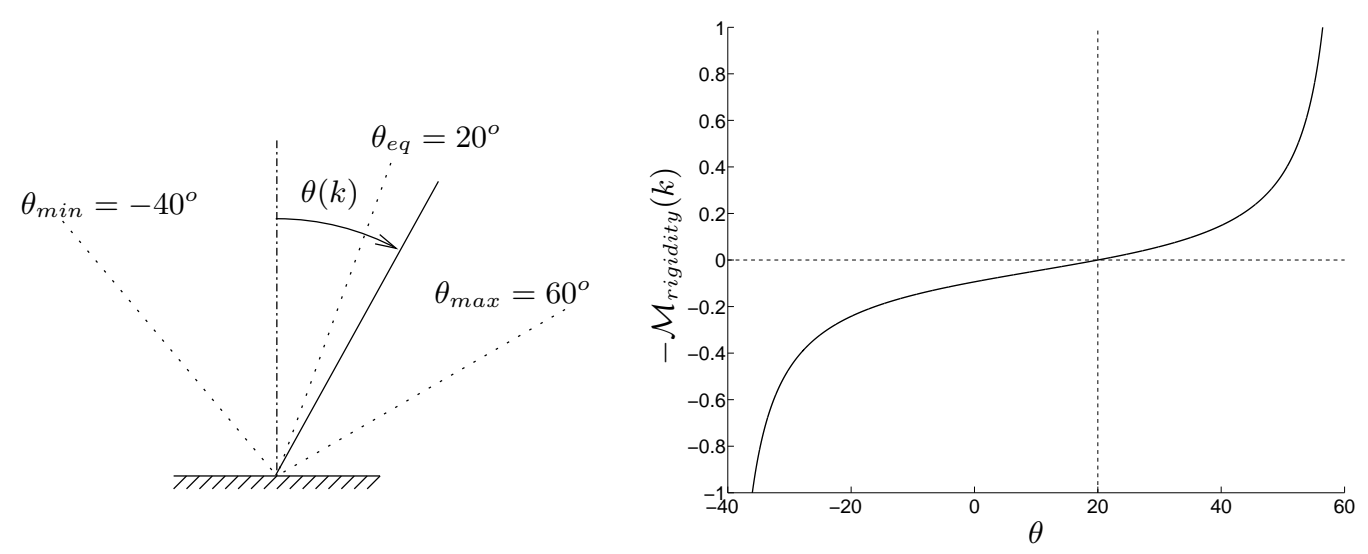

Figure 10. Moment of rigidity as a function of the angle of one reference cilium $\theta(k)$. The angle of equilibrium is chosen to be $\theta_{e q}=20^{\circ}$, and $\theta_{\min }$ and $\theta_{\max }$ are set to $-40^{\circ}$ and $60^{\circ}$.

The temporal evolution of reference cilia is parametrized by $\mathcal{S}=\left(\theta(1), \ldots, \theta\left(N_{c}\right)\right)^{T}$ where $\theta(k)$ is the angular position of the $k^{t h}$ cilium and $N_{c}$ is the number of reference elements. In the moving reference frame defined by $(\mathbf{t}, \mathbf{n})$, with $\mathbf{t}$ and $\mathbf{n}$ unit vectors tangential and normal to the reference fiber, considering that the external forces are applied in the middle of each element (and the lever arm is thus $l / 2$ ), the moments at play are listed below.

\section{Rigidity:}

$$
M_{\text {rigidity }}(k)=-K_{r} f_{1}[\theta(k)] .
$$

In this expression $f_{1}$ is a non-linear function defined as: $f_{1}(\theta)=\frac{P[\theta(k)]-P\left(\theta_{e q}\right)}{P^{\prime}\left(\theta_{e q}\right)}$, where $P=\tan (a \theta+b)$ with $a$ and $b$ constants, chosen to produce the behaviour illustrated in figure 10: $a=\pi /\left(\theta_{\max }-\theta_{\min }\right)$ and $b=-a\left(\theta_{\max }+\theta_{\min }\right) / 2$. The role of $M_{\text {rigidity }}$ is to model the structural flexibility of the hairy layer with the rigidity parameter $K_{r}$ (in [kg $\left.\mathrm{m}^{2} \mathrm{~s}^{-2}\right]$ ) of the reference elements, and to control the oscillation potential of each cilium 

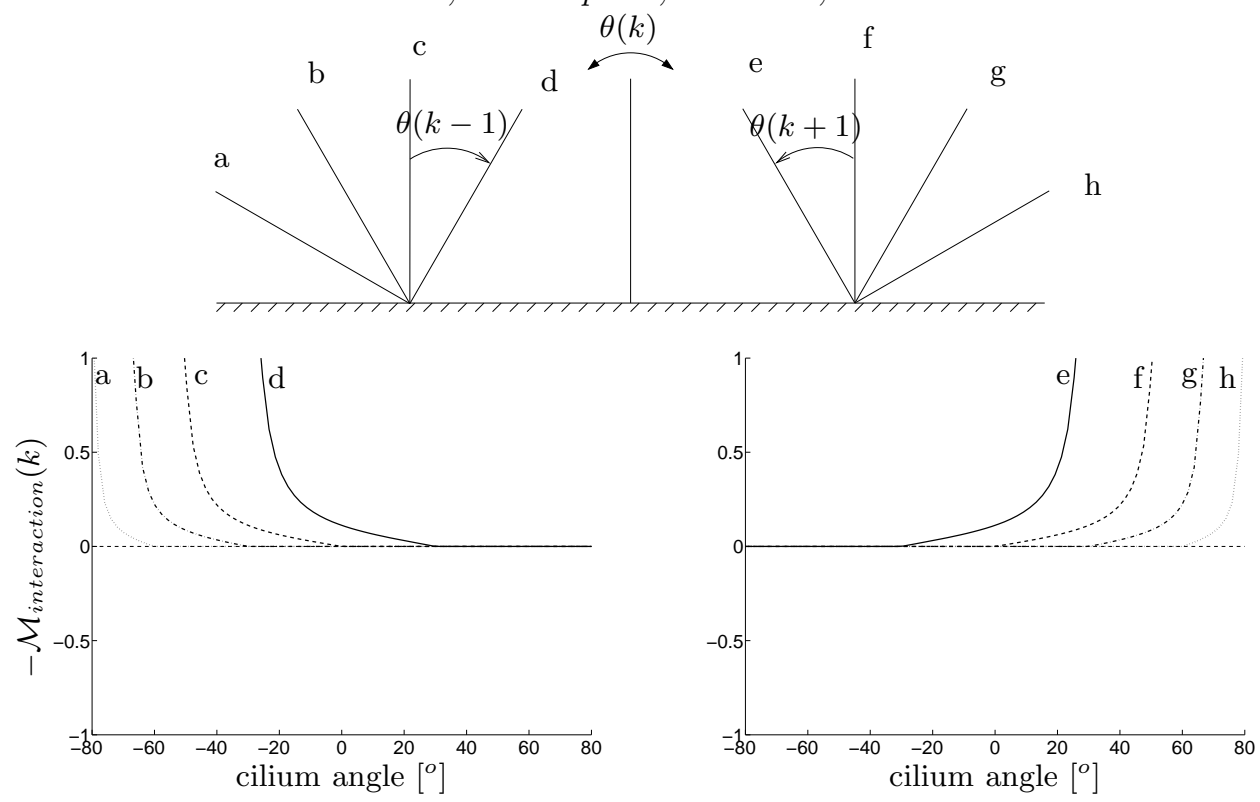

FiguRE 11. Moment of interaction as a function of the angle of one reference cilium $\theta(k)$, when the angle of the neighbouring cilium $\theta(k-1)$ is equal to $-60^{\circ},-30^{\circ}, 0^{\circ}$ and $30^{\circ}$ (left) and the angle of the neighbouring cilium $\theta(k+1)$ is equal to $-30^{\circ}, 0^{\circ}, 30^{\circ}$ and $60^{\circ}$ (right). The reference cilia interspace is taken to be equal to the length of each element.

based on the maximum deflection angles $\theta_{\min }$ and $\theta_{\max }$, and the equilibrium angle $\theta_{e q}$. Note that the slope of the curve represented on the right frame of figure 10 is $K_{r}$, i.e. $\frac{d f_{1}}{d \theta}\left(\theta_{e q}\right)=1$. This property will be useful in $\S 4$.

\section{Interaction:}

$M_{\text {interaction }}(k)$ is only activated when the reference cilia become too close to one another; this term models the interaction between two neighboring elements via a non-linear function governed by the stiffness parameter $K_{s}$ (in $\left[\mathrm{kg} \mathrm{m}^{2} \mathrm{~s}^{-2}\right]$ ), which is the same for all cilia:

$$
M_{\text {interaction }}(k)=-K_{s} f_{2}[\theta(k)] .
$$

Here, another function $f_{2}$ is introduced: $f_{2}[\theta(k)]=\tan \left\{\frac{2 l \sin \left[\theta(k)-\theta_{m}\right]}{h \cos \left(\theta_{m}\right)}\right\}$ with $\theta_{m}=$ $\frac{\theta(k)+\theta(k+1)}{2}$, where $h$ is the cilia interspace and $l$ the length (figure 9$)$. The reaction is higher when two neighbouring cilia are close to one another (figure 11).

Losses:

$$
M_{\text {losses }}(k)=-C_{l} \dot{\theta}(k) .
$$

This term is included in the model to take into account the structural losses of the oscillating reference fibers. It is taken to be a linear function of speed, with the loss parameter $C_{l}$ (in $\left[\mathrm{kg} \mathrm{m}^{2} \mathrm{~s}^{-1}\right.$ )] controlling the magnitude of the term. It is linked to the energy dissipation by plastic deformation of each hair, and to friction occurring between neighbouring elements. 
Inertia:

$$
M_{\text {inertia }}(k)=m l_{a}^{2} \ddot{\theta}(k) .
$$

This moment is related to the mass (in $[\mathrm{kg}]$ ) of the reference elements (which is the sum of the true masses of the cilia present in a reference control volume, $c f$. figure 9 ) and the length $l_{a}=l / 2$, assuming that the mass is placed in the middle of the rod.

\section{External force:}

$$
M_{\text {ext }}(k)=l_{a} F_{\text {ext }}(k) .
$$

This moment refers to the moment of the external force exerted by the fluid on each fiber, calculated by the integral of the volume force $\mathbf{F}^{h}$ over the control volume of each reference fiber $V_{\text {control }}(k)$ :

$$
F_{\text {ext }}(k)=\int_{V_{\text {control }}(k)}\left\|\mathbf{F}_{n}^{h}\right\| d V
$$

Clearly, the tangential force $\mathbf{F}_{t}^{h}$ of the fluid onto the element exerts no moment.

We recall here that $\theta(k)$ is defined around the equilibrium angle of each cilium $\theta_{e q}(k)$.

\subsection{Non-dimensional model and characteristic numbers}

To assess the physical mechanisms involved in the coupling between the fluid and the hairy layer, we define in this section non-dimensional numbers, characteristics of the fluid part, the structure part, and also numbers built on the coupled behavior of fluid and structures.

\subsubsection{Hairy layer}

The equilibrium of the system composed by all cilia is found by writing the balance of all the moments above, for all $k$. We obtain the following governing equation, for all cilia:

$$
m l_{a}^{2} \ddot{\theta}+K_{r} f_{1}(\theta)+K_{s} f_{2}(\theta)+C_{l} \dot{\theta}=l_{a} F_{\text {ext }}
$$

Along the lines of Doaré et al. (2004), we define the following frequencies: $\omega_{r}=\sqrt{\frac{K_{r}}{m l_{a}^{2}}}$, $\omega_{s}=\sqrt{\frac{K_{s}}{m l_{a}^{2}}}$ and $\omega_{l}=\frac{C_{l}}{m l_{a}^{2}}$, which are, respectively, the characteristics frequencies based on the rigidity, interaction and dissipation effects of the rods. We now render time dimensionless by introducing $t_{c}^{*}=t \omega_{r}$; the adimensional external force is $\mu_{e x t}=\frac{F_{e x t}}{m l_{a} \omega_{r}^{2}}$ and equation (3.14) in dimensionless form reads:

$$
\ddot{\theta}+\gamma \dot{\theta}+f_{1}(\theta)+\kappa f_{2}(\theta)=\mu_{e x t}\left(t_{c}^{*}\right),
$$

by introducing the parameters $\gamma=\frac{\omega_{l}}{\omega_{r}}$ and $\kappa=\frac{\omega_{s}^{2}}{\omega_{r}^{2}}$. These parameters will be useful in the following to assess the relative importance of each constitutive term of the structure model. 


\subsubsection{Fluid}

Important dimensionless numbers for the fluid are the Reynolds number, the drag coefficient $C_{d}=\frac{F_{d}}{\frac{1}{2} \rho\left\|\mathbf{U}_{\infty}\right\|^{2} S}$, with $F_{d}$ the modulus of the drag force and $S$ the projected frontal area, and the lift coefficient $C_{l}=\frac{F_{l}}{\frac{1}{2} \rho\left\|\mathbf{U}_{\infty}\right\|^{2} S}, F_{l}$ modulus of the lift force. The Strouhal number is also introduced, $S t=\frac{D}{T_{\text {flow }}|| \mathbf{U}_{\infty} \|}$, with $T_{\text {flow }}$ the characteristic period of vortex shedding. In the graphs showing the evolution of $C_{d}$ and $C_{l}$, we will employ the non-dimensional time $t_{f}^{*}=t / T_{\text {flow }}$, built on a characteristic time specific to the fluid.

To analyze the different effects of the contributions listed in $\S 3.2$, we compute the energy (per unit time) terms relative to each moment $j$ by:

$$
P_{j}=\int_{1}^{N_{c}} M_{j}(k) \dot{\theta}_{j}(k) d k .
$$

The non-dimensional energy per unit time is then defined by $P_{j}^{*}=\frac{P_{j}}{\frac{1}{2} \rho\left\|\mathbf{U}_{\infty}\right\|^{3} S}$.

\subsubsection{Fluid-structure interaction numbers}

We introduce also non-dimensional parameters built on the characteristics of both fluid and structural features, to assess the coupling between the two (De Langre 2006):

- the mass number of the whole layer $M_{\text {layer }}=\frac{\rho}{\rho_{\text {layer }}}$, where $\rho$ is the density of the fluid and $\rho_{\text {layer }}$ is the density of the whole hairy layer, i.e. hair plus fluid, given by $\rho_{\text {layer }}=\frac{m}{\mathcal{V}_{\text {control }}}+\rho(1-\phi)$

- the mass number of hair $M_{\text {hair }}=\phi M_{\text {layer }}$, characteristic of the density of hair only, compared to the fluid density.

\subsection{Numerical resolution}

The governing equations for all reference cilia, eq. (3.15), are solved using two numerical methods, explicit or implicit, depending on whether the inertial terms are included in the analysis or not. In fact, whereas an explicit approach requires necessarily a non-zero mass of the fibers to prevent divergence of the algorithm, an implicit method does not suffer from this restriction.

The explicit scheme is a Runge-Kutta method in four steps. Between two iterations $i$ and $i+1$ of the global fluid-structure resolution algorithm, the dynamics of the structure is computed explicitly at each temporal sub-iteration. Thus, the equilibrium of the cilia is found after a sufficient number of sub-iterations in time. This method is quite stable but, in the case of very small values of $m$, oscillations can appear and delay the achievement of the final equilibrium. In this case, or when $m=0$, an implicit resolution is preferred.

In the implicit approach, temporal sub-iterations are not needed and the equilibrium solution, if it exists, is found directly by minimizing the response surface of dimension $N_{c}$ formed by the sum of all the moments. A non-linear conjugate gradient method is used to find the minimum and the equilibrium is generally reached rapidly. 


\section{Frequency analysis of the dynamics of reference cilia}

We perform here a linear stability analysis of equation (3.15) by using the following decomposition:

$$
\theta=\theta_{e q}+\theta^{\prime}
$$

i.e. the angles of cilia are the sum of a steady equilibrium angle $\theta_{e q}$, and a small perturbation $\theta^{\prime}$. We consider equation (3.15) without external forcing from the fluid:

$$
\ddot{\theta}+\gamma \dot{\theta}+f_{1}(\theta)+\kappa f_{2}(\theta)=0,
$$

and focus on the natural frequency response of the system. By introducing the decomposition (4.1), and neglecting non-linear terms issued from the products of perturbations $\theta^{\prime}$, we obtain:

$$
\ddot{\theta^{\prime}}+\gamma \dot{\theta^{\prime}}+\theta^{\prime}\left[\frac{d f_{1}}{d \theta}\left(\theta_{e q}\right)\right]=0
$$

We have here neglected the contribution of the parameter of interaction $\kappa f_{2}\left(\theta_{e q}+\theta^{\prime}\right)$, assuming that the action of neighbouring cilia is negligible near equilibrium, as cilia do not get in contact with each other ( $c f$. the definition of interaction in $\S 3.2$ ). Note also that the rigidity time scale dominates the dynamics ( $c f . \S 3.3 .1)$.

Then, by definition of the moments of rigidity $(c f . \S 3.2)$ we have:

$$
\ddot{\theta}^{\prime}+\gamma \dot{\theta}^{\prime}+\theta^{\prime}=0 \text {. }
$$

We assume that the perturbations $\theta^{\prime}$ behaves as $\theta^{\prime} \sim e^{i \omega_{c}^{*} t_{c}^{*}}$, where the non-dimensional frequency is $\omega_{c}^{*}=\omega_{c} / \omega_{r}, \omega_{c}$ being the characteristic frequency of the structure model. We obtain the following equation for $\omega_{c}^{*}$ :

$$
\omega_{c}^{* 2}-i \gamma \omega_{c}^{*}-1=0,
$$

yielding $\omega_{c}^{*}=\frac{1}{2}\left[i \gamma \pm \sqrt{\left.-\gamma^{2}+4\right)}\right]$.

By using a value of $\gamma=0.05$ small compared to other terms, as it is done in $\S 6.3$, we then have $\omega_{c}^{*}= \pm 0.999+0.025 i$. The frequency of the resonant mode of the structure is $\omega_{c} \simeq \omega_{r}$, and the growth rate is negative, indicating that the system is asymptotically stable.

Strong of this result, we solve numerically equations (4.2) under the assumption that all cilia are subject to the same impulse forcing, using the parameters used in $\S 6.3$ : $K_{r}=6.75 \times 10^{-8}\left[\mathrm{~kg} \mathrm{~m}^{2} \mathrm{~s}^{-2}\right], K_{s}=1.35 \times 10^{-8}\left[\mathrm{~kg} \mathrm{~m}^{2} \mathrm{~s}^{-2}\right], C_{l}=8.21 \times 10^{-9}\left[\mathrm{~kg} \mathrm{~m}^{2}\right.$ $\left.\mathrm{s}^{-1}\right]$, with 14 reference elements of mass $m=1[\mathrm{~g}]$.

The evolution of all cilia angles is represented in the left frame of figure 12, as a function of the characteristic time $t_{c}^{*}$. We anticipate that with these settings, there are about four periods of oscillations of the structure for one period of vortex shedding of the fluid in uncontrolled conditions. The right frame of figure 12 shows the Fourier spectrum as a function of the non-dimensional frequency $\omega_{c}^{*}$. We obtain a clear peak at $\omega_{c}^{*}=1$, indicating that the dynamics of cilia is driven by $\omega_{r}$, i.e. rigidity effects, as obtained theoretically. The decrease in time $e^{-\operatorname{Im}\left(\omega_{c}^{*}\right) t_{c}^{*}}$ of cilia angles is also recovered. Here, the interaction effects between neighbouring cilia is clearly negligible, as all cilia have the same evolution and oscillate in parallel to each other.

Figure 13 shows the response of the model in the case of an impulse forcing only on the middle reference cilium (number 7). In this configuration, the contribution of the interaction parameter becomes significant, as cilia are getting closer to one another many times during the oscillations. Moreover, the oscillations in time of cilia angles (left colums) are not the same and each cilium displays a slightly different frequency content 

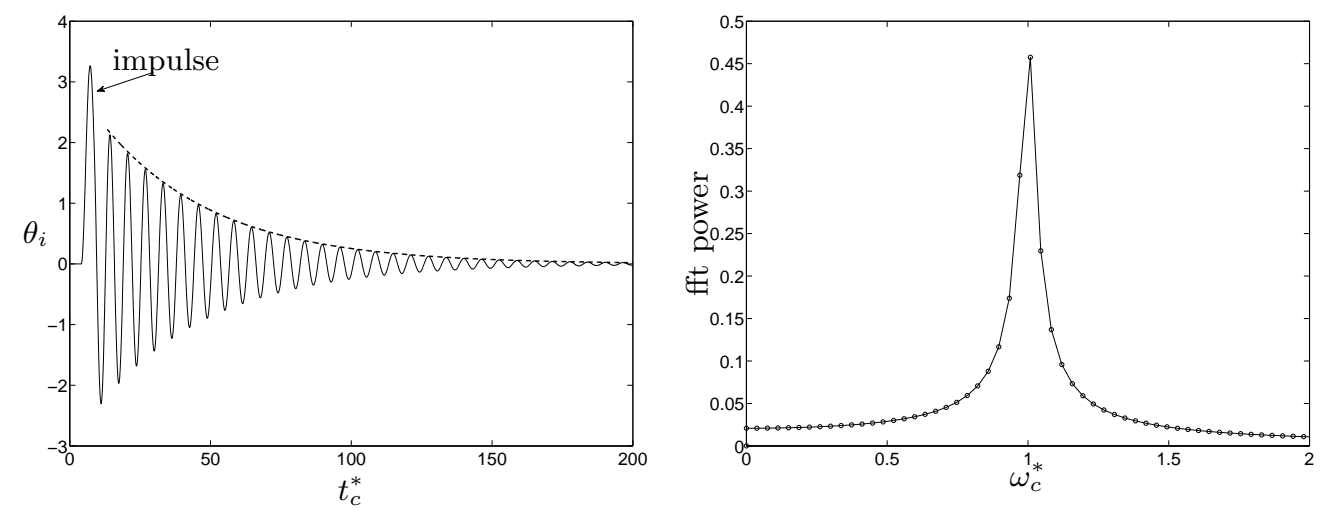

FiguRE 12. Response of the model of reference cilia without the fluid to an impulse forcing identical on all cilia. Left: Time evolution of all reference cilia angles in degrees, as a function of the non-dimensional time $t_{c}^{*}$; the dashed line represents $e^{-\operatorname{Im}\left(\omega_{c}^{*}\right) t_{c}^{*}}$ with arbitrary initial amplitude. Right: Fourier transform of the signal (for $t_{c}^{*}>16$ ) as a function of $\omega_{c}^{*}$ (the values used for rigidity, interaction and loss parameters are those of $\S 6.3)$.

(right column). The peak is modified compared to the previous case and for all cilia, the interaction parameter tends to add other harmonics to the dynamics, while the lowest frequency of the model remains dictated by rigidity.

\section{Summary of the two-way coupling}

The numerical simulation of this coupled problem corresponding to the unsteady flow past a compliant porous structure requires the simultaneous solution of structural and fluid dynamics equations. To do so, we use a partitioned procedure, staggered in time because the two parts are integrated in succession (figure 14). The coupling is efficiently performed by PALM, a software tool developed for data assimilation and meteorological applications by CERFACS (Buis et al. 2005).

$\mathcal{S}_{i}=\left(\theta_{1}, \ldots, \theta_{N_{c}}\right)_{i}^{T}$ refers to the set of state variables relative to the structure part, and $\mathbf{F}_{i}$ denotes the volume force exchanged by the different blocks. At a given iteration $i$ of the global fluid-structure procedure, the external force $\mathbf{F}_{i}$ drives the movement of the reference cilia. The equilibrium of the system is then computed by the structure solver (with the explicit or the implicit technique). Starting from the new position of each element, the packing density $\phi$ inside the hairy layer is estimated, and the reaction on the fluid by the layer is computed using the homogenized approach ( $c f . \S 3.1)$. This force is then interpolated on the fluid mesh to update the new configuration of the structure at iteration $i+1$. Then, the fluid solver computes the configuration of the flow at $i+1$. It is clear that this procedure is highly dependent on the choice of the time step of integration, since the time scale of the different constituents of the problem is not unique.

\section{Application to the open-loop control of the cylinder wake}

\subsection{No control}

We first consider the configuration of the circular cylinder at $R e=200$ without control elements (figure 15a). The time-averaged drag coefficient $\left(C_{d}=1.368\right)$ and the Strouhal number $(S t=0.20)$ are in good agreement with those obtained by He et al. (2000) 

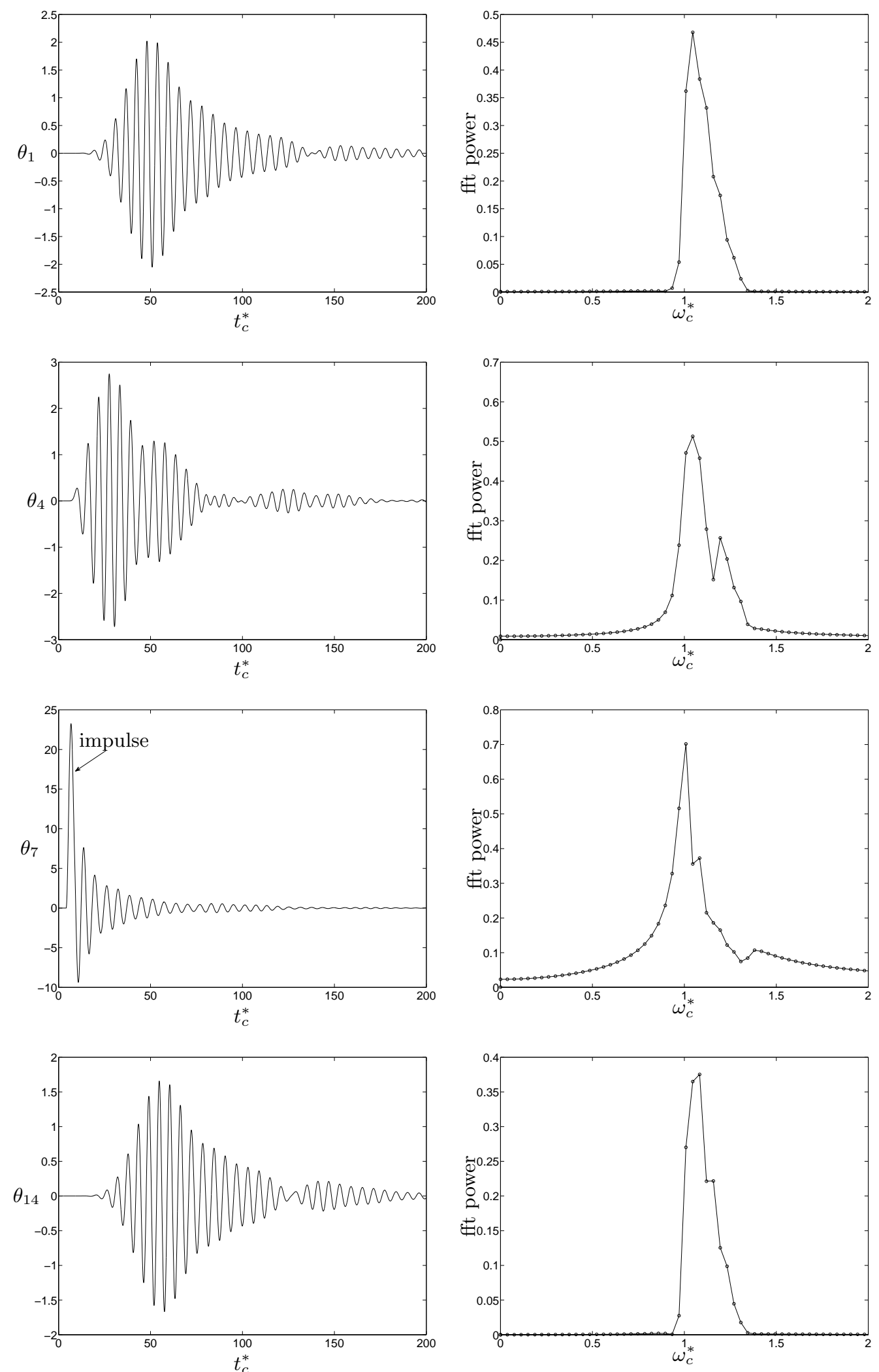

FiguRE 13. Response of the model of reference cilia without the fluid to an impulse forcing applied on cilium 7 only. Left: Time evolution of cilia number 1, 4, 7 and 14 in degrees, as a function of the non-dimensional time $t_{c}^{*}$; right: Associated Fourier transforms of the signals as functions of $\omega_{c}^{*}$. 


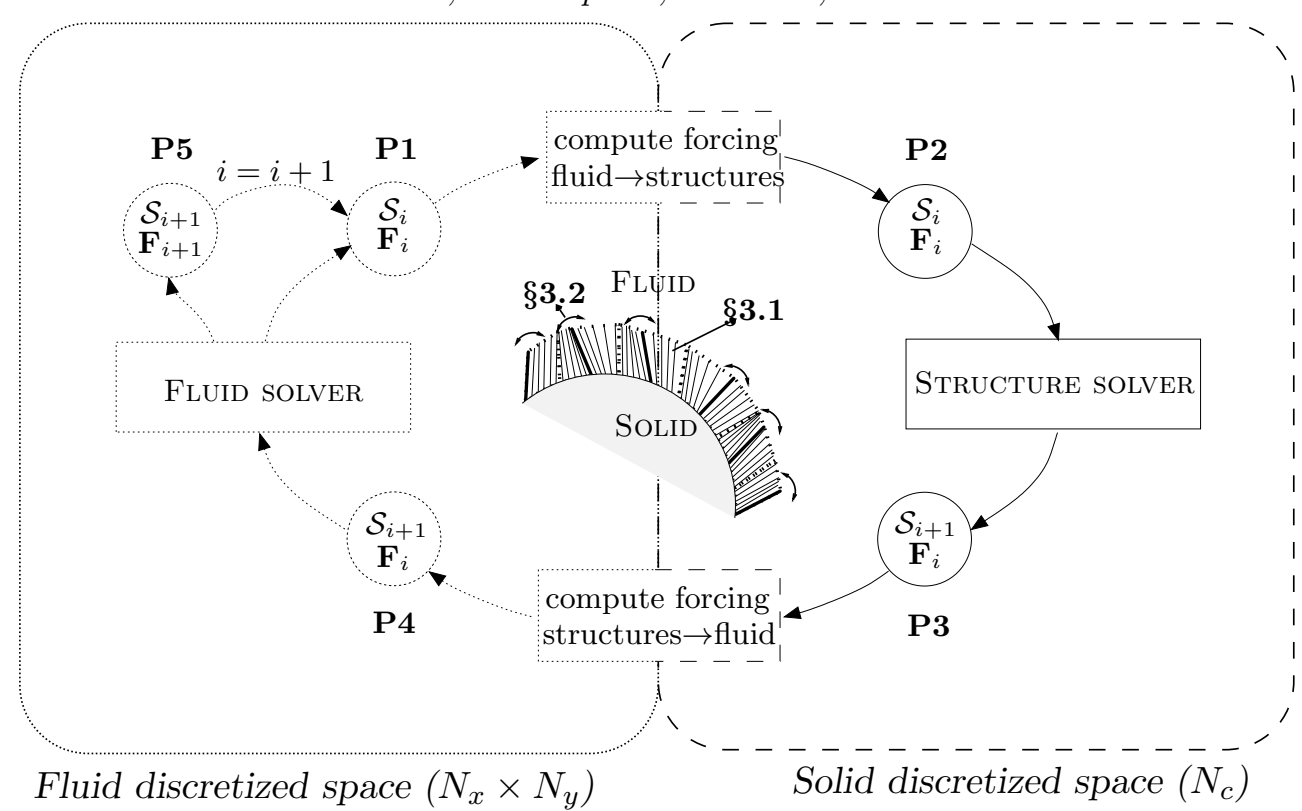

Figure 14. Two-way coupling algorithm. The successive steps are denoted $P 1$ to $P 5$; the cycle then starts over.

$\left(C_{d}=1.356\right.$ and $\left.S t=0.198\right)$ and Bergmann et al. $(2005)\left(C_{d}=1.390\right.$ and $\left.S t=0.199\right)$. The time evolution of drag and lift coefficients is illustrated in figure 22 in dashed lines.

The choice of this flow configuration at $R e=200$ is motivated by the desire to consider a flow regime dominated by a strong unsteady separated wake. Moreover, this critical Reynolds number is close to that for the onset of secondary instabilities leading to turbulence (Williamson 1996). Although three-dimensional effects start to appear before $R e=200$, the purpose of our exercise is to develop, test and assess the feasibility of the proposed passive control approach, rather than develop in-depth understanding of the controlled flow physics at low Reynolds number. Furthermore, at $R e=200$, threedimensional effects are still small enough so that excellent agreement is generally obtained by comparing Strouhal number and mean drag coefficient against three-dimensional results (Williamson 1996).

\subsection{Static test cases}

We explore the influence of the initial orientation of the hairy layer, in a static configuration, i.e. the reference control elements are fixed in their initial position, but the action of the coating on the flow is present (one-way coupling). The initial reference position of the fibers has a strong influence on the dynamics of the whole system in the case of two-way coupling, because of the definition of the equilibrium angles. This is why the position of equilibrium of the elements are studied a priori: reference cilia normal to the wall (figure 15b) or parallel to the freestream velocity (figure 15c), compared to a smooth wall (figure 15a). The circumferential extent of the hairy layer is $40 \%$ of the perimeter of the cylinder and the coating is placed symmetrically around the rear stagnation point. The cylinder has diameter $D=0.2[\mathrm{~m}]$ and the length of reference cilia is $D / 5$.

Note that only reference fibers are represented in the figure, but in between each pair of elements the layer must be seen as a continuum of more or less densily packed cilia. The packing density of the layer is fixed to 0.006 , by considering cilia diameter of 0.5 [mm] and 3 cilia per $\mathrm{cm}^{2}$. To give an idea of the packing density obtained with these 


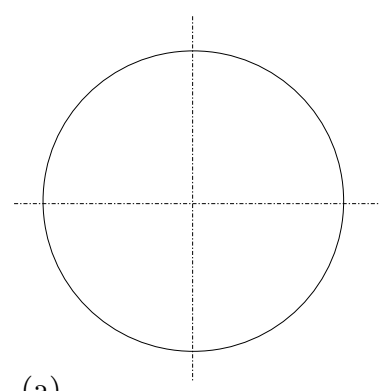

(a)

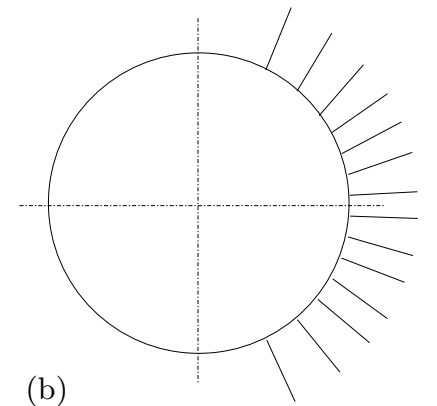

(b)

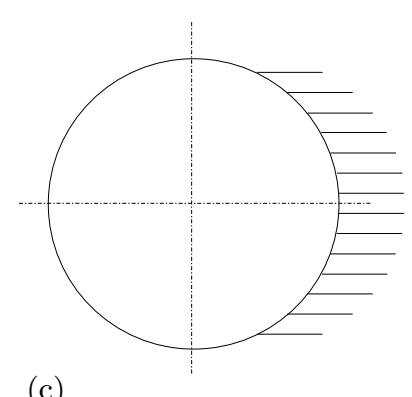

(c)

FIGURE 15. Static configurations. (a) Smooth wall. Cilia oriented (b) normal to the wall and (c) parallel to the freestream speed.

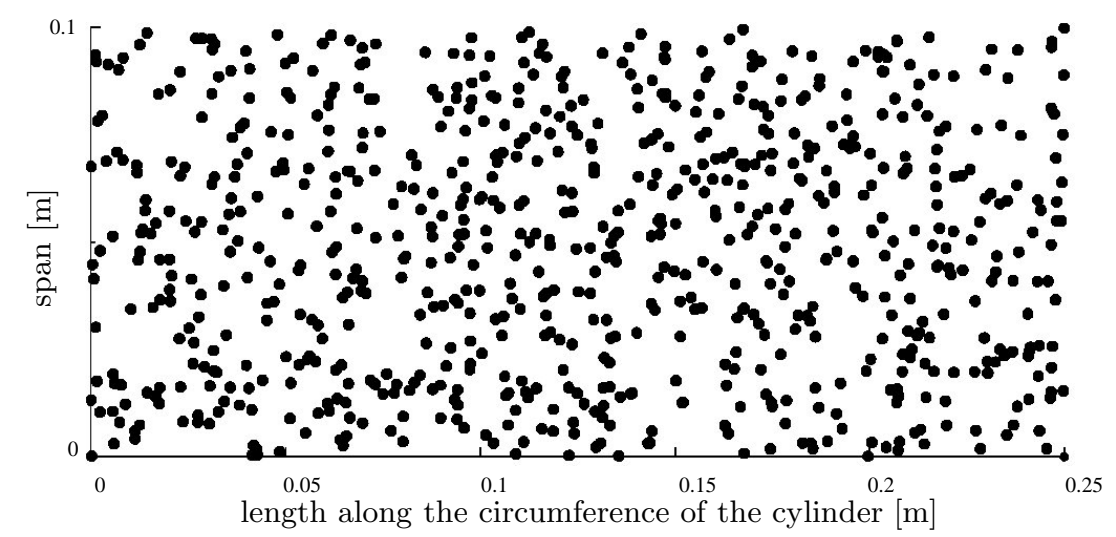

FIGURE 16. Visualisation of the density of hair (random organisation) on the unwrapped surface of a cylinder, for a packing density $\phi=0.006$, in a section of a hairy coating of length $0.25[\mathrm{~m}]$ (i.e. $40 \%$ of the perimeter of a cylinder of diameter $0.2[\mathrm{~m}]$ ) and span 0.1 [m]. 750 cilia of diameter $0.5[\mathrm{~mm}]$ are represented by black circles in this control volume of $250\left[\mathrm{~cm}^{2}\right]$.

values on a realistic hairy layer, figure 16 shows the clustering of cilia on a cylinder of diameter $0.2[\mathrm{~m}], 0.1$ [m] of span, the circumferential length of the layer being $0.25[\mathrm{~m}]$. Even for this low packing density, we can intuitively expect a relatively high resistance offered by the layer slowing down locally the oncoming flow.

Figure 17 shows the drag and lift coefficients obtained for the three configurations of figure 15, functions of the non-dimensional time $t_{f}^{*}$ defined in $§ 3.3 .2$. We obtain a drastic drag increase $(130 \%)$ with an orientation normal to the wall (case (b) of figure 15), compared to the smooth wall case. This is due to the parasitic drag created by the reference elements placed at the extremities of the layer, generating a strong resistance to the oncoming flow. Conversely an orientation of the hairy layer parallel to the flow shows the opposite trend, with a drag reduction of $4.7 \%$ and oscillations of the lift coefficient of moderate amplitude. This orientation tends to render the cylinder similar to an airfoil, possibly guiding the flow in the proximity of the trailing edge. In the following, we will study the dynamics of the hairy layer around this equilibrium state.

\subsection{Results of the two-way coupling}

Once the Reynolds number and the equilibrium orientation of the layer are fixed, the space of control parameters is rather large:

- five parameters of the dynamical model which specify the structural properties of 

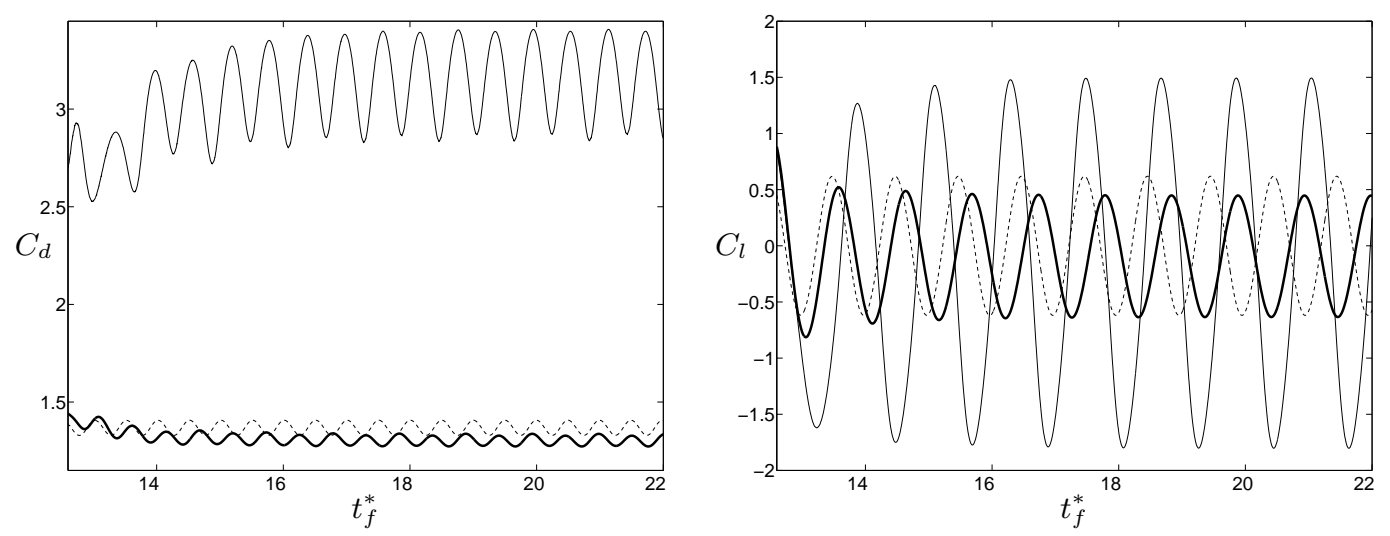

Figure 17. Aerodynamic coefficients corresponding to the static layers of figure 15. Left: Drag coefficient. Right: Lift coefficient. Dashed lines correspond to the configuration a) with smooth walls; thin solid lines correspond to the configuration b) with an orientation normal to the wall; bold lines correspond to the configuration c) with an orientation parallel to the flow.

the coating in terms of rigidity $\left(K_{r}\right)$ stiffness $\left(K_{s}\right)$, losses $\left(C_{l}\right)$, number and mass of reference pillars $(m)$,

- one parameter for the homogenized model controlling the density of hair (set by the diameter $d_{h}$ of the fiber and the number of hair per $\mathrm{cm}^{2}$ ),

- two more parameters describing the location of the control zone in the physical space: the position of the hairy layer on the wall cylinder and its thickness, i.e. the length of the fibers.

After an exploratory study at $R e=200$, and according to the modal response analysis of $\S 4$, we have found a set of five efficient parameters for the model of the layer: $K_{r}=$ $6.75 \times 10^{-8}\left[\mathrm{~kg} \mathrm{~m}^{2} \mathrm{~s}^{-2}\right], K_{s}=1.35 \times 10^{-8}\left[\mathrm{~kg} \mathrm{~m}^{2} \mathrm{~s}^{-2}\right], C_{l}=8.21 \times 10^{-9}\left[\mathrm{~kg} \mathrm{~m}^{2} \mathrm{~s}^{-1}\right]$, with 14 reference cilia of mass $m=1[\mathrm{~g}]$ on a cylinder of diameter $D=0.2[\mathrm{~m}]$. The packing density of the layer is fixed at 0.006 when the hair layer is at rest, corresponding to real cilia diameter of $0.5[\mathrm{~mm}]$ and a packing of 3 cilia per $\mathrm{cm}^{2}$ (cf. figure 16). Finally, the control zone is located over the downstream part of the cylinder $\left(\alpha_{c}=72^{\circ}\right.$ on figure 18). The thickness of the layer is set to $l / D=0.2$, which is a typical value of feathers length for a wide range of birds. The initial position and orientation of the hairy layer at the instant of the activation of control is set as in figure 15c (reference angle of $\theta_{e q}=0^{\circ}$ ), with the fibers initially parallel to the freestream velocity. Note that the configuration with the feathers initially flush to the surface has also been tested. However it has caused numerical problems because of the very fine near-wall grids required to correctly capture the coupling between fluid and structures. Also, for many of the cases tested, the reference elements remained stuck to the wall, with no interesting results in terms of drag reduction. Thus, we focus on the effect produced on a separated flow region by a set of feathers which are already popped up from the surface.

Table 1 presents the relevant non-dimensional numbers corresponding to this case. The value of the parameter $\gamma$ is small compared to the other terms of equation (3.15), indicating that the characteristic time of the waves related to the structural dissipation $\omega_{l}^{-1}$ is much larger than $\omega_{r}^{-1}$. The value of the ratio $\kappa / \gamma^{2}=\omega_{s}^{2} / \omega_{l}^{2}$ is here equal to 80 . Thus $\omega_{l}^{-1}$ is also larger than $\omega_{s}^{-1}$, characteristic time of the oscillations due to the interaction between reference cilia. The force of the fluid can dominate the other terms 


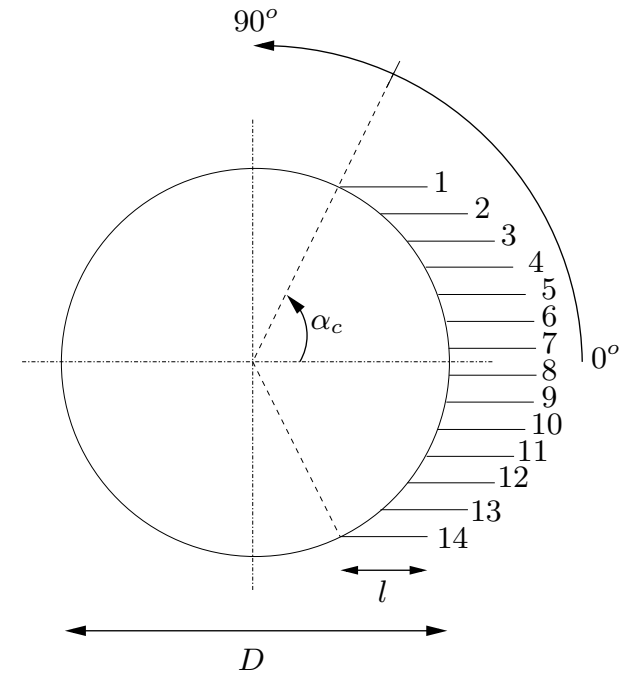

Figure 18. Position of the control zone on the wall cylinder and reference cilia numbers. The angular parameter $\alpha_{c}$ determines the extent of the layer with respect to the rear stagnation point.

$\frac{\operatorname{Re} \| \gamma\left[\frac{\text { losses }}{\text { rigidity }}\right]\left|\kappa\left[\frac{\text { interaction }}{\text { rigidity }}\right]\right| \mu_{\text {ext }}^{\max }\left[\frac{\text { fluid forcing }}{\text { rigidity }}\right]}{200 \quad \| \quad 0.05} \mid$

TABLE 1. Non-dimensional numbers for the fluid and the reference cilia; $\mu_{e x t}^{\max }$ is the maximum amplitude of the non-dimensional force exerted by the fluid onto the structures, in the fully developed regime.

of equation (3.15), as indicated by the maximum value of $\mu_{e x t}^{\max }$ in Table 1 , as large as nine times the rigidity restoring force.

As indicated in $\S 4$, the waves propagating in the hairy layer are mainly controlled by the fluid force and the rigidity parameter, with smaller contributions due to the interaction between reference cilia and losses. This will be discussed further down in the context of figure 19 .

The mass number of the whole layer, fluid and structures, is equal to $M_{\text {layer }}=0.49$, indicating that the layer has comparable inertia to the fluid. In fact, since $\rho_{\text {layer }} \simeq 2 \rho$, the layer would sink when immersed in the fluid, like typical birds' feathers. The low value of the mass number of hair alone, $M_{\text {hair }}=0.003$, suggests that the mass of fluid displaced by the control elements is very small. By taking $\rho=1.2\left[\mathrm{~kg} \mathrm{~m}^{-3}\right]$, it is obtained $\rho_{\text {hair }}=\frac{\rho}{M_{\text {hair }}}=400\left[\mathrm{~kg} \mathrm{~m}^{-3}\right]$, which is of the same order of magnitude as the keratin fiber of birds' feathers (Barone \& Schmidt (2006) report a volume of $890\left[\mathrm{~kg} \mathrm{~m}^{-3}\right]$ ).

Starting the simulation with the hairy coating from a steady symmetric regime (no von Kármán shedding) would yield a transient regime to an unknown developed state without direct link to the oscillating state described in the literature. Here, the hairy layer is activated starting from a fully established unsteady regime (i.e. the configuration without coating), and the dynamics of the fluid and structure part is monitored together with the drag and lift coefficients $C_{d}$ and $C_{l}$. 

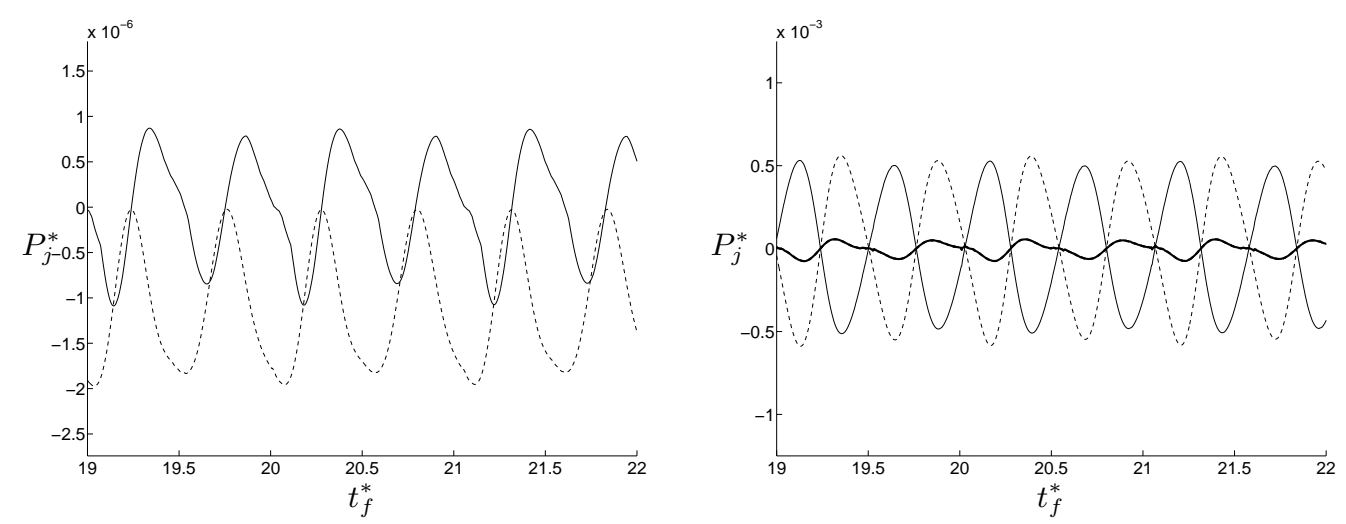

FiguRE 19. Power terms relative to the structural control parameters. Left: Minor contributions: structural losses (dashed line) and interaction parameter (continuous line) Right: Major contributions : rigidity parameter (dashed line) and external forcing (plain line). $d E_{c s} / d t$ is also plotted in bold lines. It is strictly equal to the sum of all terms and its time average vanishes.

With these settings the different terms in the energy balance are represented in figure 19; the balance is satisfied for both implicit and explicit methods $\left(\sum_{j} P_{j}=\frac{d E_{c s}}{d t}\right)$ presented in $\S 3.4$. The structural losses of the hairy layer and the interaction parameter (figure 19 left frame) have a contribution which is about two orders of magnitude smaller than the contributions of the other terms. The opposition to the external forcing is mainly done by the fibers' rigidity term (figure 19 right frame). These time evolutions are related to the oscillating motion of reference cilia as represented in figure 20. Only the evolution of the first seven cilia of the top of the layer are shown, since the seven others evolve symmetrically with respect to the horizontal axis. The time origin of the graphs corresponds to the instant when the control is activated. After an initial transient, all cilia reach an established regime beating with a period $T_{\text {cilia }}=1.03 T_{\text {flow }}$. The cilium placed at the extremity of the layer hardly moves and the amplitudes of the angles increase when the reference cilia approach the horizontal axis of symmetry. The biggest amplitudes, around 50 degrees, are observed for the cilia closest to the axis during the transient regime when the two-way coupling is turned on and the layer adapts to the flow.

Figure 21 shows a snapshot of the contours of vorticity together with the position of reference cilia. According to the time evolutions of the angles, the hairy layer tends to orient itself as a function of the position of the near-wall recirculating zone and the sign of the near-wall vorticity, alternatively positive and negative over one period of shedding. Movie 1, available with the online version of the paper, provides a better appreciation of this behaviour.

The drag and lift coefficients corresponding to this case are displayed in figure 22 . When the control is activated, the drag coefficient is reduced by $11.5 \%$ at the end of the simulation, when the controlled flow reaches a fully developed regime, and the amplitude of the fluctuations is reduced by half. Moreover there is a reduction of $33 \%$ on the amplitude of oscillations of the lift coefficient. The wake is stabilized by this passive actuation on the flow, as shown by the effect on the mean and fluctuating global coefficients.

From figure 22 (right frame), we estimate the Strouhal number $S t=0.200$ associated to the vortex shedding. With the presence of the hairy layer, $S t$ is reduced to the value of 0.193 , and both structure and fluid are beating at this frequency. Thus, the shedding 

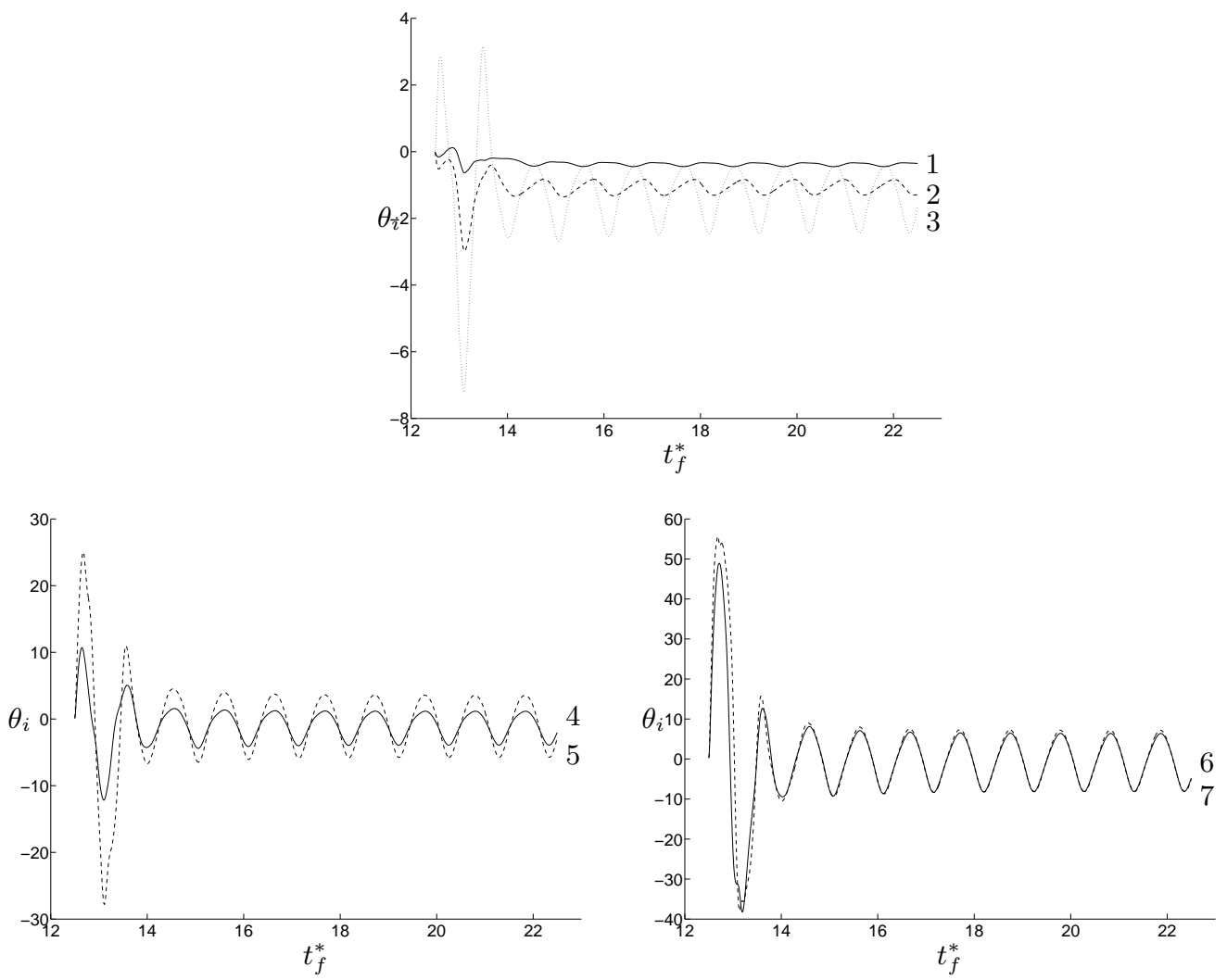

FigURE 20. Time evolution of the angles $\alpha$ (in degrees) with respect to the horizontal axis of the seven reference cilia shown in figure 18.
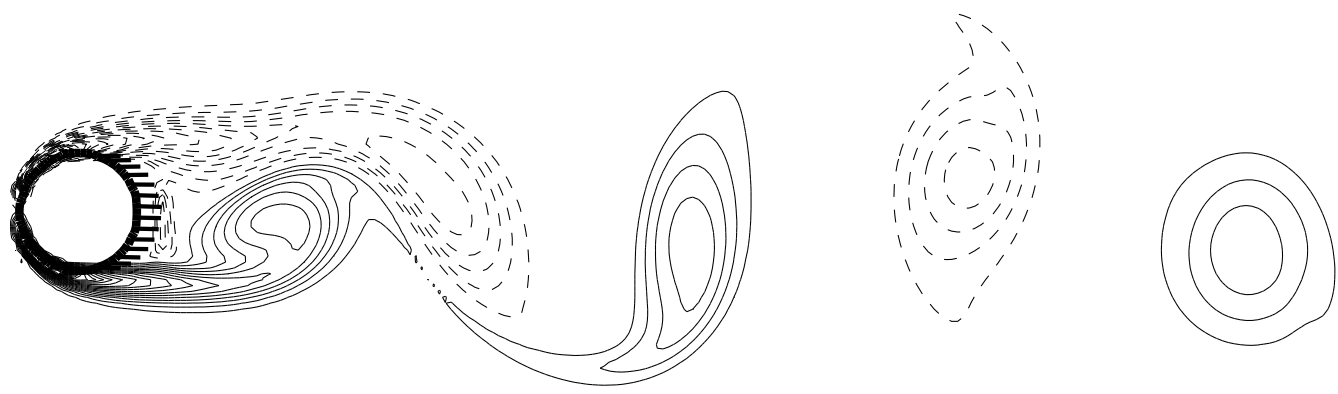

FIGURE 21. Instantaneous contours of vorticity in the presence of beating cilia (continuous lines: positive, dashed lines: negative). The time evolution of the vorticity field together with the fluctuating lift and drag is shown in movie 1, available with the online version of the paper.

frequency deviates from its natural frequency as it is generally found when an oscillating fluid instability is coupled to a vibrating structure (De Langre 2006; Py et al. 2006). The frequency common to the fluid and the hairy layer is not however that of the resonant mode of the structure, which is about 4 times larger ( $c f$. $\S 4$ ). Hence, the structures lock onto a frequency very close to the natural frequency of oscillations of the fluid system. 

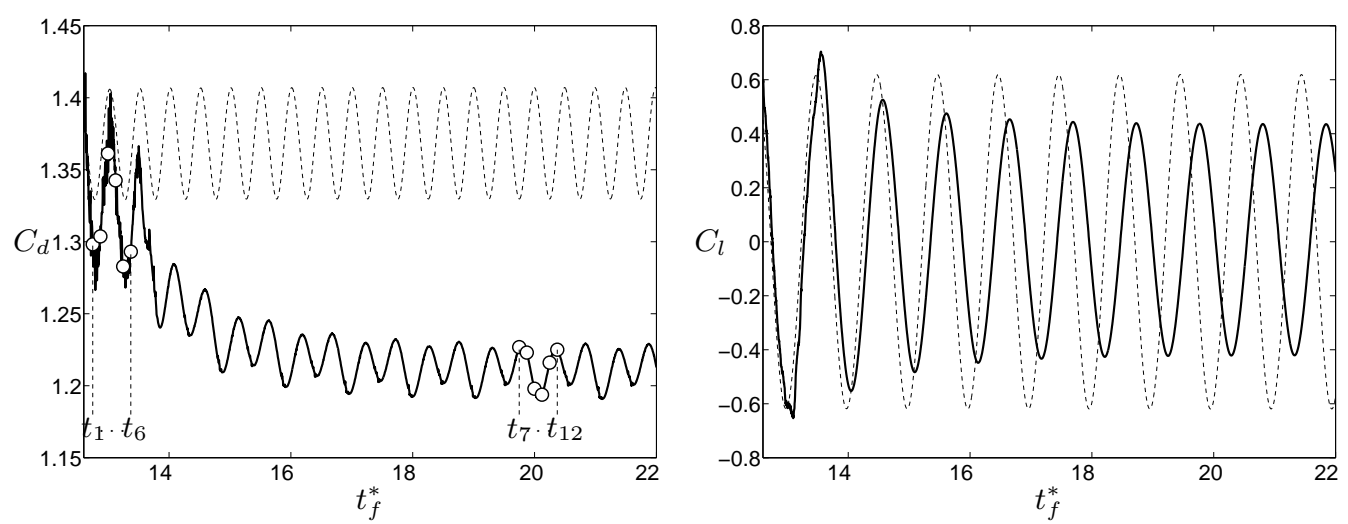

FiguRE 22. Aerodynamical coefficients without control (dashed lines) and when control is on (bold). Left: Drag coefficient. Right: Lift coefficient. The instants relative to the snapshots of figure 25 and 26 are represented in white circles (equally spaced in time).

\subsection{Physical analysis of the control}

The influence of the control on the mean flow is displayed on figures 23 and 24 . To extract the action of the control on the mean velocity field we focus on the mean quantity $\frac{<\mathbf{U}^{c}>-<\mathbf{U}>}{\left\|\mathbf{U}_{\infty}\right\|}$, defined by the non-dimensional difference between the local timeaveraged velocity, with and without control. A few observations apply:

- near the wall ( $x \leqslant D / 2$ where $x$ is measured from the rear stagnation point on the cylinder's wall), the vortices attached to the cylinder have a lower circulation when the hairy layer is present: indeed, the difference between the velocity fields with and without control shows an action opposed to the flow inside the separation bubble;

- further downstream $(D / 2 \leqslant x \leqslant 2 D)$, the field on the figure indicates a deceleration of the flow: there are large amplitude disparities from right to left at the center of the figure, and consequently, a modification of the vortex shedding process;

- as a consequence of the two previous observations, there is a connection between the near-wall region and the far-field zone, to satisfy the divergence-free condition. The topological boundary between the two regions takes the form of a saddle point located about $D / 2$ downstream of the wall.

The effect of the control on the mean pressure field is shown in figure 24, with contours of the non-dimensional quantity $\frac{\left\langle P^{c}>-<P>\right.}{\rho\left\|\mathbf{U}_{\infty}\right\|^{2}}$. As a consequence of the modifications to the velocity fields, the differences between the controlled and uncontrolled cases are mainly concentrated on a high-pressure zone, whose maximum coincides with the saddle point.

The pressure field is modified by the control and pressure is larger downstream of the cylinder, while the "dead water" region is enlarged. A similar control effect has been observed by Pastoor et al. (2008), described as a delay of the appearance of asymmetries in the flow, thus suppressing the effects of the wake instability. This is directly related to the decrease of the drag coefficient observed.

Note that a comparison of the modes issued from a proper orthogonal decomposition of the fluctuating velocity and pressure fields, with control and without control, shows small phase shifts between the two cases, linked to the modification of the Strouhal number. The POD modes are not shown for the sake of brevity. The modes' energy for the controlled case is slightly lower than in the case without control but the spatial 


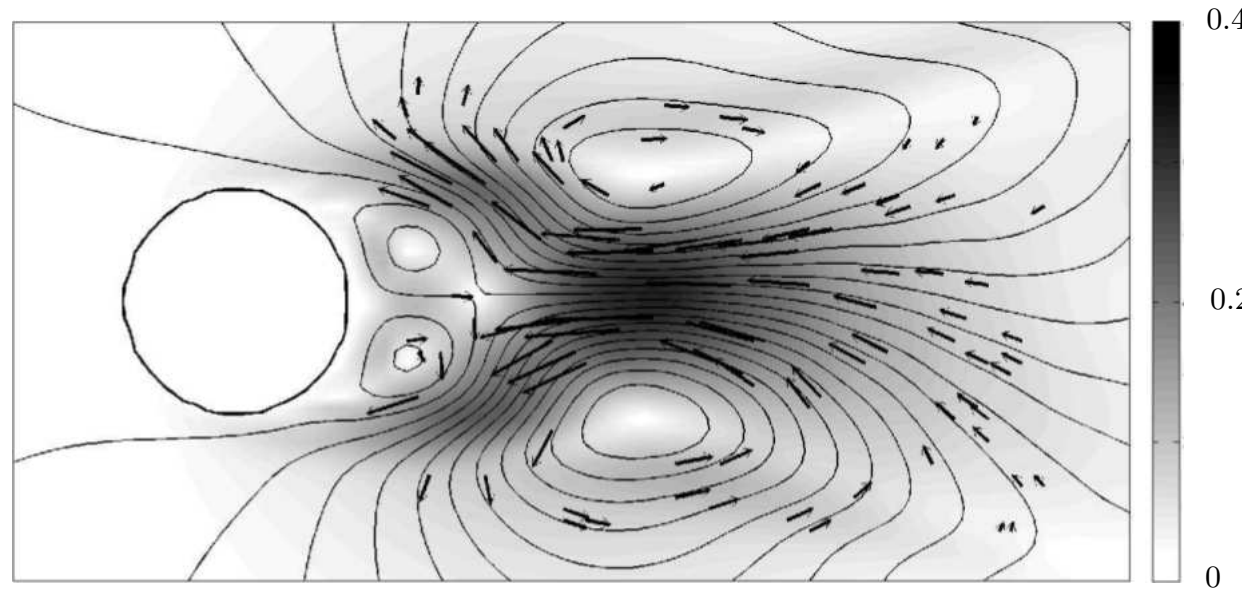

FIGURE 23. Isocontours of the streamfunction of the vector field $\frac{<\mathbf{U}^{c}>-<\mathbf{U}>}{\left\|\mathbf{U}_{\infty}\right\|}$ in plain lines with arrows. Background contours are coloured by the norm of the vector.

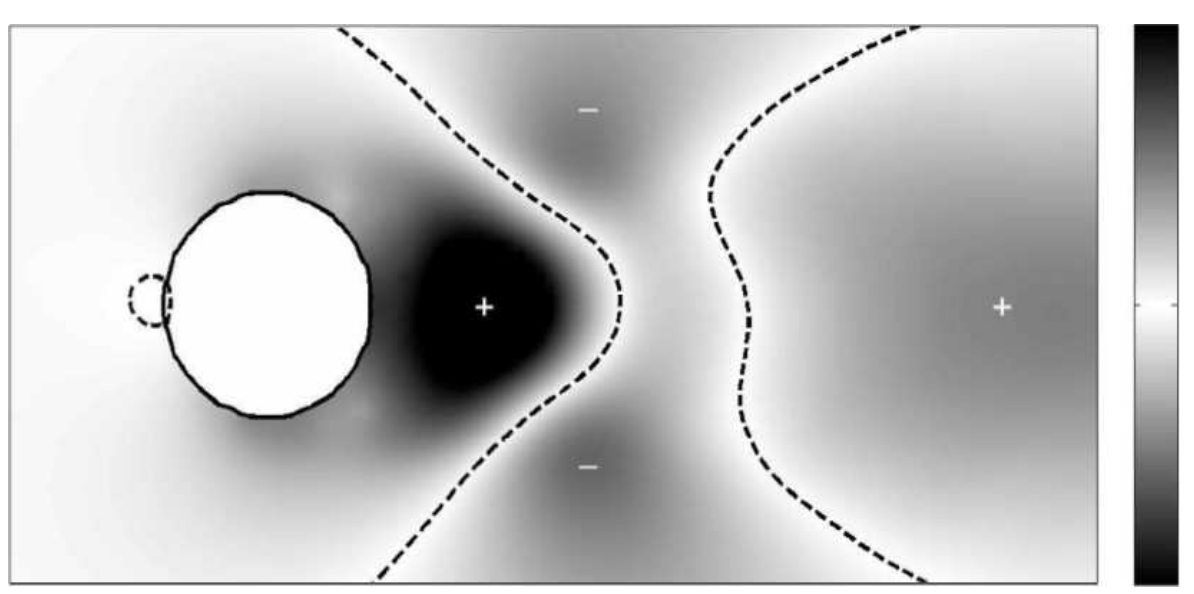

Figure 24. Contours of the non-dimensional scalar field $\frac{\left\langle P^{c}>-<P>\right.}{\rho\left\|\mathbf{U}_{\infty}\right\|^{2}}$. The zero isocontours are shown in black dashed lines; white plus and minus signs refer to positive and negative zones.

organisation of the modes is very similar for the two cases, indicating that the effect of the hairy layer is primarily on the mean flow. Figure 25 presents snapshots of the near-wall flow (contours of vertical velocity), together with the instantaneous position of the reference cilia, at 6 time instants within the initial transient state ( $c f$. figure 22).

From time $t_{1}$ to $t_{6}$ the angles of the cilia during this transient go from their minimum to their maximum values (from $-40^{\circ}$ to $55^{\circ}$ for the cilia placed near the axis of symmetry). At particular time instants $\left(t_{2}\right.$ and $t_{5}$ for instance), oriented bundles of reference cilia can be seen in the lower region for snapshot $t_{2}$ and in the upper region for snapshot $t_{5}$. In these configurations when the reference cilia are getting closer to one another, the density of cilia increases ( $c f$. the homogenized model). The magnitude of the force is a function of the density of cilia, and there is the tendency for stronger forcing when two reference cilia are close to one another. In other words, when the reverse flow at the back of the 

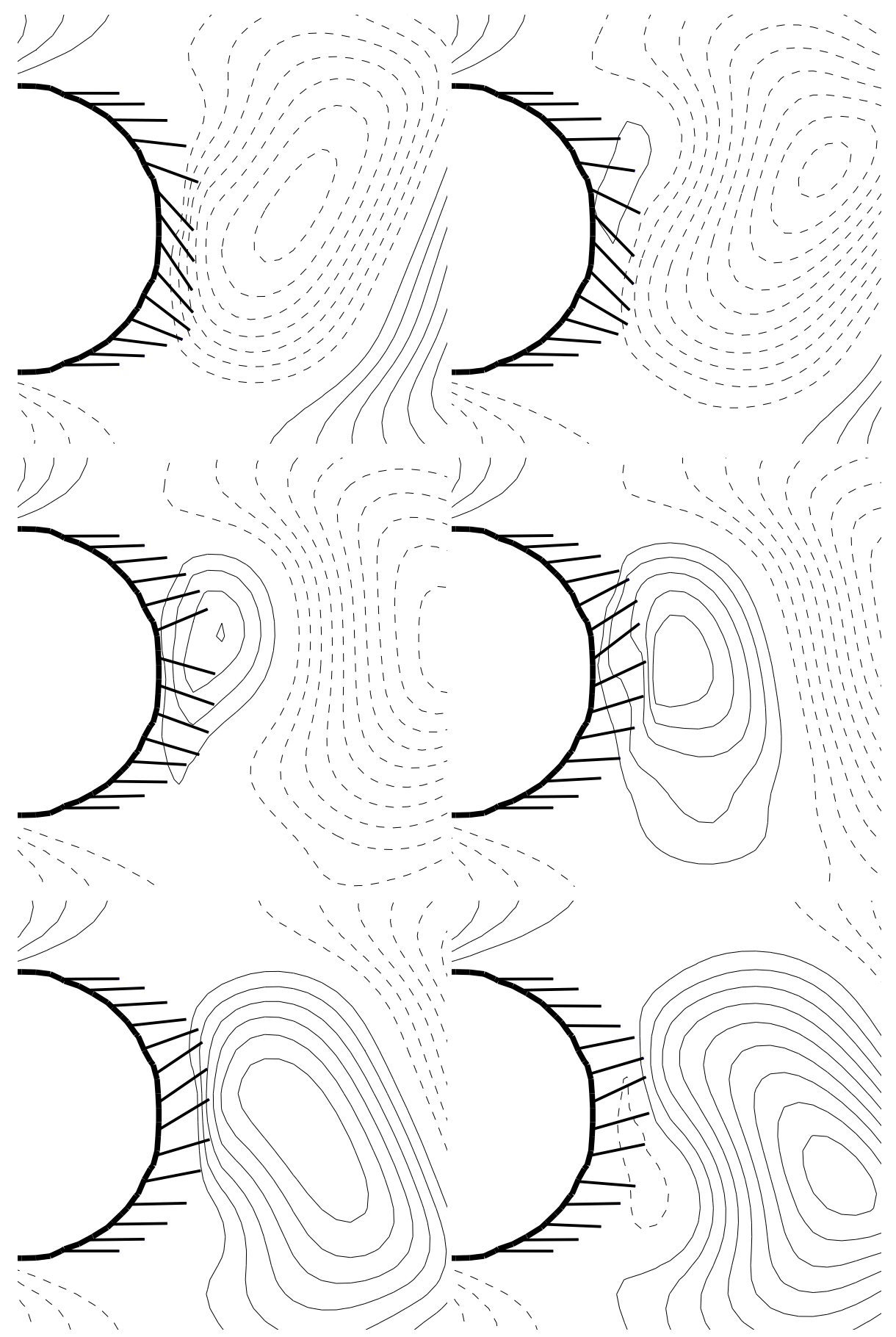

Figure 25. Motion of the reference cilia at the six time instants $t_{1}$ to $t_{6}$ shown in figure 22, arranged as in a comic strip (from left to right, and top to bottom). Snapshots of vertical velocity contours are displayed in the background (plain lines: positive, dashed lines: negative). The movement of the reference fibers is shown with the evolution of the vertical velocity in time in movie 2 , available with the online version of the paper. 


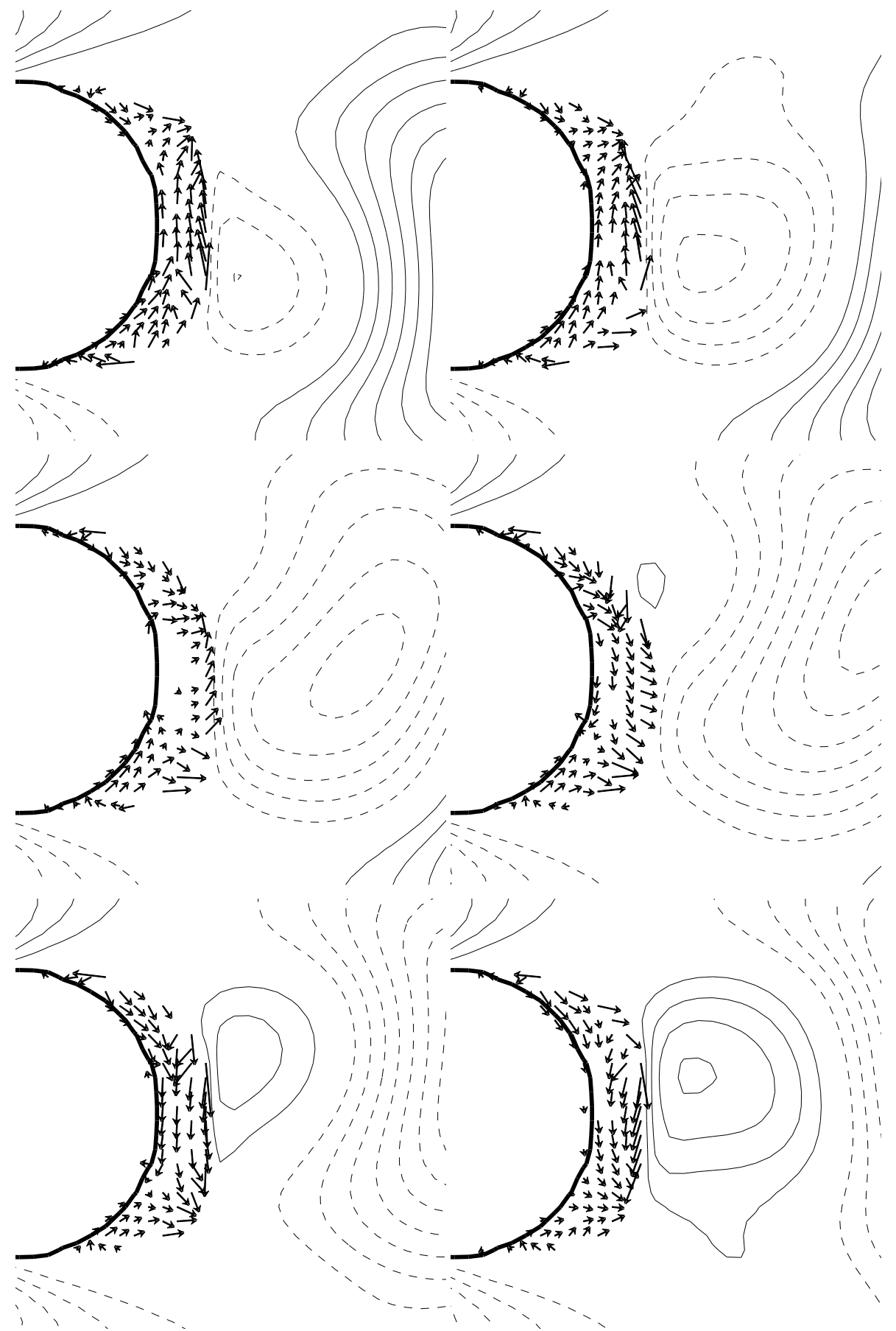

FiguRE 26. Force exerted by the hairy layer on the fluid at the six time instants $t_{7}$ to $t_{12}$ shown in figure 22, arranged as in a comic strip (from left to right, and top to bottom). Snapshots of vertical velocity contours (plain lines: positive, dashed lines: negative). The evolution in time of the force field is shown with the evolution of the vertical velocity in time in movie 2, available with the online version of the paper. 


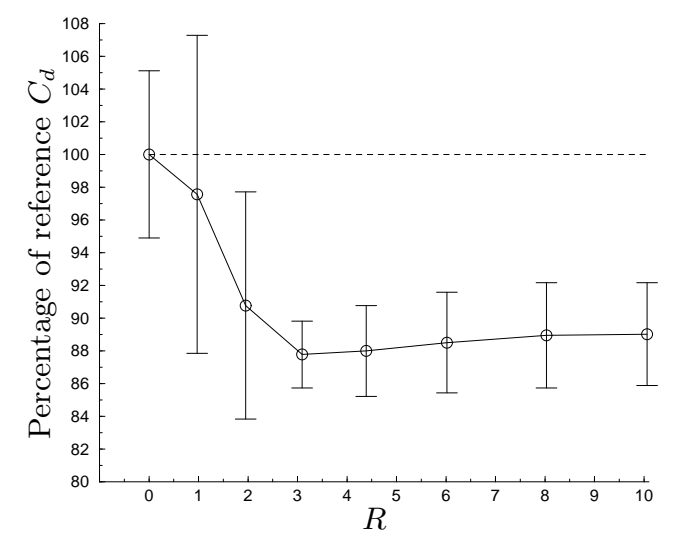

FiguRE 27. Effect of the resonant mode of the structure model on the drag, by varying the rigidity parameter from $K_{r}=3.7 \times 10^{-9}\left[\mathrm{~kg} \mathrm{~m}^{2} \mathrm{~s}^{-2}\right]\left(T_{\text {flow }} / T_{\text {cilia }}=1\right)$ to $K_{r}=3.6 \times 10^{-7}$ $\left(T_{\text {flow }} / T_{\text {cilia }}=10\right)$. The drag coefficient is plotted as a function of $R=\omega_{c} \frac{T_{\text {flow }}}{2 \pi}$; vertical bars indicate the amplitude of the temporal fluctuations of the drag coefficient.

cylinder is compressing the coating, the control force naturally increases to counteract this effect. This appears to be linked to the wings of birds, whose feathers pop-up during landing. Together with the movement of reference cilia, the control force (exerced by the hairy layer on the fluid) is shown at six other time instants corresponding to a fully established controlled regime ( $c f$. figure 22). As time progresses, the force tends to have an orientation opposed to the velocity field induced by the presence of the vortices about to be shed: when the flow is going from top to bottom $\left(t_{8}\right)$ the force is opposed ( $c f$. figure 26 ). The same counteracting behaviour occurs at snapshot $t_{11}$, when the flow goes from bottom to top (movie 2 provides a better appreciation of this effect). This is related to a lock-in mechanism mentioned in $§ 6.3$. Indeed, the recirculating zone is forced to lock onto a frequency slightly different from the natural one, and the instability is mitigated.

Thus, the hairy layer acts like a self-adapting actuator, passive (as no input energy is required) and leading to substantial improvements in the lift and drag forces.

\subsection{Parametric study}

We explore in this section a part of the space of control parameters described in $\S 6.3$, by considering variations around the values tested so far. The influence of the packing density is analysed first and we investigate afterwards the effect of the spatial properties of the hairy coating. We first test the influence of the resonant frequency of the structure model by varying the rigidity parameter. The loss parameter $C_{l}$ is fixed at the low value of $8.21 \times 10^{-9}\left[\mathrm{~kg} \mathrm{~m} \mathrm{~m}^{-1}\right]$, in order to minimize this effect compared to the others. Indeed, a very dissipative layer would slow the flow too much by converting the flow kinetic energy into structural losses, an effect which does not appear to characterize an effective hairy coating. The interaction parameter is fixed at the value of $1.35 \times 10^{-8}[\mathrm{~kg}$ $\left.\mathrm{m}^{2} \mathrm{~s}^{-2}\right]$, so that the frequency response due to this parameter is that described in $\S 4$. The number, mass and length of reference cilia are the same as in $\S 6.3$, i.e. 14 reference cilia of mass $m=1[\mathrm{~g}]$ and length $l=0.04[\mathrm{~m}]$, to consider a density of the layer close to that of real hairy coatings.

\subsubsection{Resonant mode of the hairy layer}

The rigidity parameter is varied to modulate the compliance of the layer, thus modifying the frequency of the waves propagating within the set of reference cilia. Figure 27 


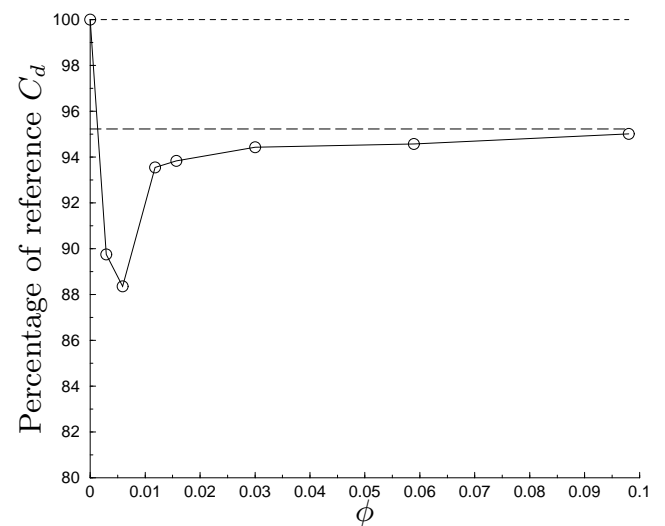

FIGURE 28. Effect of the inner density of the hairy layer $\phi$ on the drag. The two horizontal lines indicate the values of the time-averaged drag coefficient for the uncontrolled case (top dashed line) and for the case of the static configuration displayed in figure 15c (long dashed line).

shows the influence on the mean drag of the resonant mode of the reference cilia model. It is found that the best performances in terms of mean drag coefficient are obtained for a value of $R=\omega_{c} \frac{T_{\text {flow }}}{2 \pi}=3$; also, the amplitude of the temporal oscillations of the drag coefficient (vertical bars in the figure) are minimized around $R=3$. For lower values of $R$, large fluctuations in $C_{d}$ are indicated by the bars, as the structure model is close to its natural resonant frequency. In this case, the variations of reference cilia angles become large and the resulting force on the fluid induces strong fluctuations, which make the flow more unstable. Note that the value of $K_{r}=6.75 \times 10^{-8}\left[\mathrm{~kg} \mathrm{~m}^{2} \mathrm{~s}^{-2}\right]$ chosen in $\S 6.3$ corresponds to $R=4.38$.

\subsubsection{Packing density of the hairy layer}

Figure 28 shows the evolution of the drag as the packing density $\phi$ is varied. In practice, we keep the same diameter of cilia $(0.5[\mathrm{~mm}])$ and change the number per $\mathrm{cm}^{2}$ from 1.5 to 50 (see figure 16 for reference). It is found that after the value of $\phi=0.006$, the drag is increased as the hairy layer tends to behave like a static porous layer $\left(\left(C_{d}=1.3\right.\right.$ for the configuration c) of $\$ 6.2$ ). To obtain significant gains in performances, the packing density needs to be kept low enough, so that a hydroelastic-like surface wave develops on the coating. An optimal value is found in this configuration at about $\phi=0.006$.

\subsubsection{Position of the hairy layer}

The influence of the position of the hairy layer is investigated through the variation of the parameter $\alpha_{c}$, characterizing the extremities of the control zone (figure 18). The left frame of figure 29 shows the drag coefficient obtained when $\alpha_{c}$ varies from $0^{\circ}$ (no control) to $90^{\circ}$ (the whole downstream portion of the cylinder is covered with the coating). It is found that for $\alpha_{c}$ greater than $36^{\circ}$, the results change very little. However, the drag increases for values of $\alpha_{c}$ between $0^{\circ}$ and $36^{\circ}$, indicating that the control zone has to be at least larger or equal to the recirculating zones shown in figure 26.

\subsubsection{Thickness of the coating}

The influence of the thickness of the hairy layer is illustrated on the right frame of figure 29. The parameter $l / D$ is varied from 0 (no control) to 0.5. Beyond this value the layer is thicker than the cylinder radius, which is the characteristic dimension of flow 

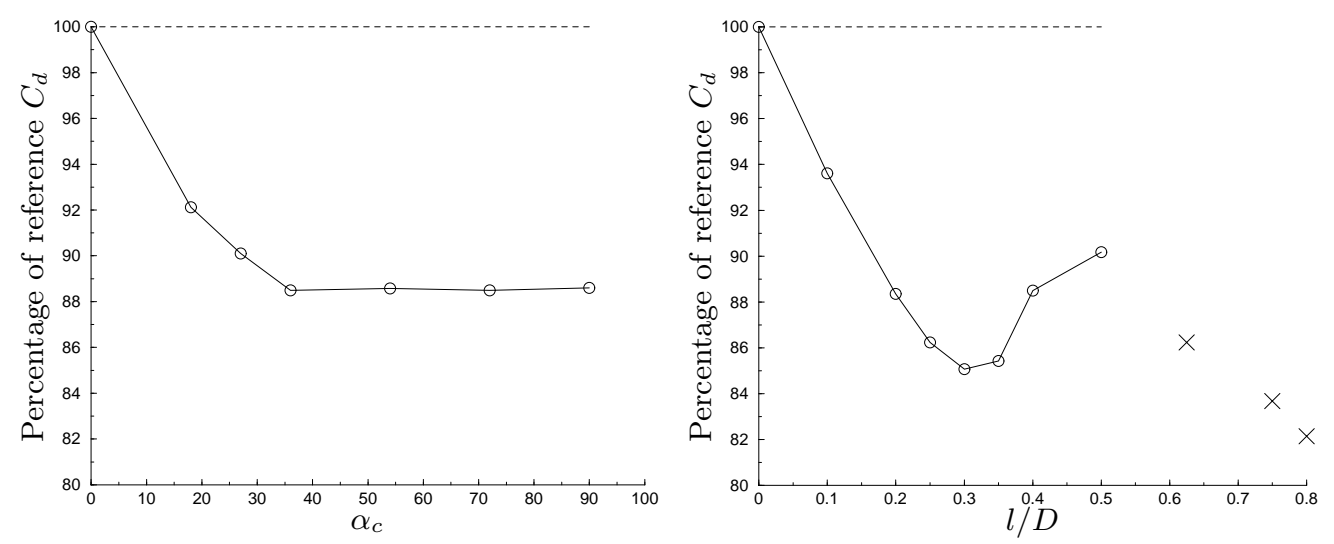

FIGURE 29. Effect of the position of the control zone on the drag. Left: drag coefficient as a function of the angular position $\alpha_{c}$ of the control zone. Right: drag coefficient as a function of the hairy layer thickness $l / D$.

features near the wall, and a model based upon rigid reference cilia with a single degree of freedom does not appear to be suitable.

A local minimum is found for $l / D=0.3$ which corresponds to a drag reduction of $15 \%$ associated to a drastic reduction of the lift fluctuations of $44 \%$. The drag increases with $l / D$, until $l / D$ reaches the value of 0.5 , which is the length of the recirculation bubble at this Reynolds number. The points beyond $l / D=0.5$ are shown simply to give an idea of the possibilities of flow manipulation provided by the use of longer and rigid elements. Even if the model is not designed for this case, drag appears to be even further reduced after $l / D=0.5$; however this is a probably shape-adaptation effect, and it would be more realistic to allow for each individual element to bend, to mimick the behaviour of real flexible cilia.

\section{Conclusions and perspectives}

We have studied the passive control of flow separation using a fuzzy coating. To test and analyse this concept of flow manipulation, a numerical methodology has been developed and used to solve a fluid-structure interaction problem. A partitioned approach has been set up to solve non-linear coupled equations for the fluid and solid parts, based on a coupler previously used in Dauptain et al. (2008). Besides the numerical aspects, an original model for a hairy layer has been proposed and tested. Directly inspired from the natural properties of birds feathers, i.e. porosity, anisotropy and compliance, it is easily extendable to other configurations.

It is found numerically that such a coating is capable of increasing global aerodynamics performances of an immersed body, by adapting to the separated flow. The effects of the control are of order one, and appear on the mean pressure and velocity fields. The topology of the flow is changed in the vicinity of the wall but also further downstream, thus modifying the vortex shedding process, and positively affecting the pressure distribution, to reduce lift fluctuations and drag. An effect of the control is to mitigate the flow instability by an elongation of the reciculation bubble, a phenomenon termed "direct opposition control" in Pastoor et al. (2008). The analysis of the forces produced by the hairy layer on the fluid shows that the coating naturally adapts to counteract the near-wall separated flow: a lock-in effect appears in which the coating synchronizes onto a frequency which is close to the natural frequency of the fluid system. 
A set of efficient control parameters is found, by linking physical mechanisms to the characteristic dimensions of the system. The thickness of the hairy layer has to match the size of the recirculation bubble close to the wall. Its packing density must reach a minimum value of 0.006 to obtain the largest drag reduction. With this combination of parameters, the drag reduction obtained is about $15 \%$ and the fluctuations in lift coefficient are reduced by $44 \%$.

The central argument in favour of this type of control is that it does not require any input of energy, and this makes it very attractive for industrial applications, compared to active control devices which are often very demanding in terms of power requirements, and thus expensive to implement on realistic conditions. The use of this type of passive actuators to control flow separation appears to be promising, particularly for applications which do not require strong constraints on the lift force (road or underwater vehicles, MAVs, etc.).

Additional work on different configurations is needed to study the robustness of this passive control technique. In particular, it is of great importance to assess the behavior of the coating under turbulent conditions. Concerning realistic applications, the presence of the environment is also very important: weather conditions (rain, cold, etc.) or the alteration of the coating characteristics over time are typical issues to take into account to ensure a robust control procedure.

Three aspects are primarily concerned for the perspectives. The first one concerns the application of this numerical tool to a more complex configuration at higher Reynolds number on an airfoil, to approach real applications or birds' flight conditions.

The second perspective is to add a third spatial component to the model of the hairy layer, and to the fluid domain. We can reasonably think that the general conclusions would not be qualitatively affected by doing so. Indeed, as far as the structure model is concerned, adding a degree of freedom does not add any conceptual difficulty and should not alter dramatically the physics of the control, at least for flows - such as the present one - which present one dominating stream direction.

Finally, to get closer to realistic layers, and to explore the possibilities allowed by the employment of long fibers, it is interesting to let the reference elements bend, by adding several layers of rigid elements linked to each other through articulated connections, along the lines of Lindström \& Uesaka (2007). We expect the bending to change the results, but probably not the general conclusions in terms of the effect of the passive control. Indeed, the ability to adapt to the flow will be increased, and we could anticipate even better results in terms of performance enhancement.

The authors gratefully acknowledge the support provided by EU Marie-Curie EST grant, through the FLUBIO project (MEST-CT-2005-020228). Donald L. Koch is thanked for useful advices on the estimate of the drag past a cluster of cylinders.

\section{REFERENCES}

Barone, J.R. \& Schmidt, W.F. 2006 Effect of formic acid exposure on keratin fiber derived from poultry feather biomass. Bioresource Technology 97 (2), 233-242.

Bechert, D.W. \& Bartenwerfer, M. 1989 The viscous flow on surfaces with longitudinal ribs. Journal of Fluid Mechanics 206, 105-129.

Bechert, D.W., Bruse, M., Hage, W. \& Meyer, R. 1997 Biological surfaces and their technological application-laboratory and in flight experiments on drag reduction and separation control. 28th AIAA Fluid Dynamics Conf., Snowmass Village, CO .

Benjamin, T.B. 1960 Effects of a flexible boundary on hydro-dynamic stability. Journal of Fluid Mechanics 9, 513-532. 
Bergmann, M., Cordier, L. \& Brancher, J.P. 2005 Optimal rotary control of the cylinder wake using proper orthogonal decomposition reduced-order model. Physics of Fluids 17, 097101.

BrüCKER, C., SpAtZ, J. \& SChrÖDER, W. 2005 Feasability study of wall shear stress imaging using microstructured surfaces with flexible micropillars. Experiments in Fluids 39 (2), 464-474.

Bruneau, C.H. \& Mortazavi, I. 2008 Numerical modelling and passive flow control using porous media. Computers and Fluids 37 (5), 488-498.

Buis, S., Piacentini, A. \& DÉClat, D. 2005 PALM: a computational framework for assembling high-performance computing applications. Concurrency and Computation: Practice and Experience 18 (2), 231-245.

Carpenter, P.W. \& Garrad, A.D. 1985 The hydrodynamic stability of flow over Kramertype compliant surfaces. I: Tollmien-Schlichting instabilities. Journal of Fluid Mechanics 155, 465-510.

Carpenter, P.W. \& Garrad, A.D. 1986 The hydrodynamic stability of flow over Kramertype compliant surfaces. II: Flow-induced surface instabilities. Journal of Fluid Mechanics 170, 199-232.

Dauptain, A., Favier, J. \& Bottaro, A. 2008 Hydrodynamics of cilary propulsion. Journal of Fluids and Structures 24 (8), 1156-1165.

De LANGRe, E. 2006 Frequency lock-in is caused by coupled-mode flutter. Journal of Fluids and Structures 22 (6-7), 783-791.

De Langre, E. 2008 Effects of wind on plants. Annual Review of Fluid Mechanics 40, 141-168.

Doaré, O., Moulia, B. \& De Langre, E. 2004 Effect of Plant Interaction on Wind-Induced Crop Motion. Journal of Biomechanical Engineering 126, 146.

Fish, F.E. \& Lauder, G.V. 2006 Passive and active flow control by swimming fishes and mammals. Annual Review of Fluid Mechanics 38, 193-224.

Gad-El-Hak, M. 2000 Flow Control: Passive, Active, and Reactive Flow Management. Cambridge University Press.

Gray, J. \& SAnd, A. 1936 The Locomotory Rhythm of the Dogfish (Scyllium canicula). Journal of Experimental Biology 13 (2), 200-209.

Grosse, S. \& SchröDER, W. 2008 Mean wall-shear stress measurements using the micro-pillar shear-stress sensor MPS 3. Measurement Science and Technology 19 (1), 015403.

He, J.W., Glowinski, R., Metcalfe, R., Nordlander, A. \& Periaux, J. 2000 Active control and drag optimization for flow past a circular cylinder i. Oscillatory cylinder rotation. Journal of Computational Physics 163 (1), 83-117.

Hepffner, J., Bottaro, A. \& J., FAVier 2008 Mechanisms of non-modal energy amplification in channel flow between compliant walls. Journal of Fluid Mechanics submitted.

Howells, I. D. 1998 Drag on fixed beds of fibres in slow flow. Journal of Fluid Mechanics 355, 163-192.

Koch, D. L. \& LADD, A. J. C. 1997 Moderate Reynolds number flows through periodic and random arrays of aligned cylinders. Journal of Fluid Mechanics 349, 31-66.

LANDAHL, M.T. 1962 On the stability of a laminar incompressible boundary layer over a flexible surface. Journal of Fluid Mechanics 13, 609-632.

Lindström, S.B. \& Uesaka, T. 2007 Simulation of the motion of flexible fibers in viscous fluid flow. Physics of Fluids 19, 113307.

Luchini, P., Manzo, F. \& Pozzi, A. 1991 Resistance of a grooved surface to parallel flow and cross-flow. Journal of Fluid Mechanics 228, 87-109.

Menta, R.D. \& Pallis, J.M. 2001 The aerodynamics of a tennis ball. Sports Engineering 4 (4), $177-189$.

Meyer, R., Hage, W., Bechert, D.W., Schatz, M., Knacke, T. \& Thiele, F. 2007 Separation control by self-activated movable flaps. AIAA Journal 45 (1), 191-199.

Pastoor, M., Henning, L., Noack, B.R., King, R. \& Tadmor, G. 2008 Feedback shear layer control for bluff body drag reduction. Journal of Fluid Mechanics 608, 161-196.

Peskin, C. S. 2002 The immersed boundary method. Acta Numerica 11, 479-517.

Py, C., De Langre, E. \& Moulia, B. 2006 A frequency lock-in mechanism in the interaction between wind and crop canopies. Journal of Fluid Mechanics 568, 425-449. 
Steele, C., Jones, R. \& Leaney, P.G. 2006 Tennis ball fuzziness: assessing textile surface roughness using digital imaging. Measurement Science and Technology 17 (6), 1446-1455.

Sukhodolova, T., Sukhodolov, A. \& Engelhardt, C. 2004 A study of turbulent flow structure in a partly vegetated river reach. River Flow 2004.

van Nierop, E.A., Alben, S. \& Brenner, M.P. 2008 How Bumps on Whale Flippers Delay Stall: An Aerodynamic Model. Physical Review Letters 100 (5), 54502.

Viswanath, P.R. 2002 Aircraft viscous drag reduction using riblets. Progress in Aerospace Sciences 38 (6-7), 571-600.

Williamson, C.H.K. 1996 Vortex dynamics in the cylinder wake. Annual Review of Fluid Mechanics 28 (1), 477-539.

Xu, J., Maxey, M.R. \& Karniadakis, G.E.M. 2002 Numerical simulation of turbulent drag reduction using micro-bubbles. Journal of Fluid Mechanics 468, 271-281. 